LPTENS-02/60, IHES/P/02/83

FIAN/TD-15/02, ITEP/TH-56/02

\title{
Complex Curve of the Two Matrix Model and its Tau-function
}

\author{
Vladimir A. Kazakov, ${ }^{1 *}$ and Andrei Marshakov ${ }^{123 \circ}$ \\ ${ }^{1}$ Laboratoire de Physique Théorique de l'École Normale Supérieure, ${ }^{\star}$ \\ 24 rue Lhomond, 75231 Paris CEDEX, France \\ ${ }^{2}$ Institut des Hautes Études Scientifiques, \\ 35 route de Chartres, 91440 Bures-sur-Yvette, France \\ 3 Theory Department, P.N.Lebedev Physics Institute and \\ Institute of Theoretical and Experimental Physics, \\ Moscow, Russia
}

\begin{abstract}
We study the hermitian and normal two matrix models in planar approximation for an arbitrary number of eigenvalue supports. Its planar graph interpretation is given. The study reveals a general structure of the underlying analytic complex curve, different from the hyperelliptic curve of the one matrix model. The matrix model quantities are expressed through the periods of meromorphic generating differential on this curve and the partition function of the multiple support solution, as a function of filling numbers and coefficients of the matrix potential, is shown to be a quasiclassical tau-function. The relation to $\mathcal{N}=1$ supersymmetric Yang-Mills theories is discussed. A general class of solvable multi-matrix models with tree-like interactions is considered.
\end{abstract}

November, 2002

* kazakov@lpt.ens.fr

○ mars@lpi.ru, mars@gate.itep.ru

* Unité mixte de Recherche 8549 du Centre National de la Recherche Scientifique et de l'Ecole Normale Supérieure et à l'Université de Paris-Sud

$\diamond$ Permanent address 


\section{Introduction}

Among the vast scope of matrix ensembles a distinguished role together with the integrals over a single matrix is played by two matrix models - the ensemble of two matrices, usually with the simplest possible interaction between them [1]. Being still simple integrable systems (like in the one matrix case their partition function is a tau-function of Toda lattice hierarchy [2]), these models possess already a richer mathematical structure than one matrix models and thus give rise to more applications. The two matrix model was proposed and studied in [3] as an important solvable example of a new class of statisticalmechanical systems: spins with nearest neighbor interaction on planar graphs (Ising spins in this case). Its multi-critical generalization, in the spirit of the one matrix multi-critical points [4], leads to the complete picture of $(p, q)$-critical points in two-dimensional gravity [5], 6] 1t. It appears also in the context of two-dimensional Laplacian growth [8], [9], [10] demonstrating some hidden parallels between all these problems.

Matrix models have been the first physical example when the partition functions were directly related to tau-functions of integrable systems [2]. The relation between the (quantum) partition functions and tau-functions of (classical) integrable systems is still rather intriguing, it appears to be much more universal than one could expect before. The same sort of integrable structure like in the matrix models and/or the topological string theories has been found [11] in the context of Seiberg-Witten theories [12] or $\mathcal{N}=2$ supersymmetric gauge theories in four dimensions.

The similarity between matrix models and supersymmetric gauge theories based on the similarity of their integrable structures was noticed long ago [13]. However, in multidimensional supersymmetric gauge theories, apart from a recent example [14, one mostly observed the quasiclassical limit of integrable hierarchies (see 15 for details and references). It means that the prepotentials of gauge theories are described rather in terms of the quasiclassical tau-functions or tau-functions of universal Whitham hierarchy [16], than the tau-functions of dispersionfull hierarchies.

The overwhelming part of the work on the matrix models in the planar (large $N$ ) limit concerned the so called one cut case, when the eigenvalues form a single support distribution, though a few interesting papers on the multi-support distributions were written in the past, especially [17] (see also [18]), where the relation with the hyperelliptic curves was revealed. All these papers were devoted to the one matrix model.

Recently, a new interest to the multi-cut solutions was born, due to the papers [19], where the effective superpotentials in the $\mathcal{N}=1$ supersymmetric gauge theories were related to the matrix models. The multi-cut solution corresponds there to the breaking of the gauge group into a few subgroups. It was also proposed in [19] to "fill" by the

1 A similar picture arises in the generalized one matrix model in external field [7], and it is not surprising that the multi-support solutions in these models are also related. 
eigenvalues not only the minima of the matrix potential, but also the maxima, the situation missed in the matrix model literature, due to the obvious absence of stability of such configurations. However, from the mathematical point of view, especially from the point of view of the analytic curve description such filling appears to be admissible and even having some nice physical applications. These aspects of the multi-cut solution were further developed in recent papers [20], [21], [22].

In this paper we are going to study the multi-support solutions in the two matrix model. As in the one matrix model case, these solutions can be formulated in terms of geometry of the underlying complex curve endowed with a generating differential and a quasiclassical tau-function, of the type proposed in [16]. We will write down explicitly the equation for the complex curve in the two matrix model and define the partition function in the planar limit as a quasiclassical tau-function. We will also study an important degenerate case of real potentials and demonstrate the consistency of the tau-function approach with the planar graph expansion (in terms of a multi-phase Ising system on the graphs) for the multi-support solution in the two matrix model. Our method of construction of the algebraic curve of the two matrix model is powerful enough to be generalizable to the more general multi-matrix models with the so called tree-like interactions of matrices.

The two matrix model with the simplest interaction between matrices is known in the literature in two, superficially different, forms. The partition function of the normal matrix model (NMM) $)^{3}$ of two commuting complex conjugated $N \times N$ matrices $\Phi, \Phi^{\dagger}:\left[\Phi, \Phi^{\dagger}\right]=0$ is defined as follows:

$$
\mathcal{Z}_{N}[t, \bar{t}]=\int \mathcal{D} \Phi \mathcal{D} \Phi^{\dagger} e^{-\operatorname{Tr} \Phi \Phi^{\dagger}+2 \operatorname{Re} \operatorname{Tr} W(\Phi)} .
$$

where the harmonic part of the potential $V\left(\Phi, \Phi^{\dagger}\right)=-\Phi \Phi^{\dagger}+2 \operatorname{Re} W(\Phi)$ is parameterized as $W(\Phi)=\sum_{k=1}^{K} t_{k} \Phi^{k}$. Going to the eigenvalues $\Phi=\operatorname{diag}\left(z_{1}, \ldots, z_{N}\right)$ we obtain l $_{\text {: }}$

$$
\mathcal{Z}_{N}^{(N M M)}[t, \bar{t}]=\int \prod_{m=1}^{N}\left(d^{2} z_{m} e^{-z_{m} \bar{z}_{m}+2 \operatorname{Re} W\left(z_{m}\right)}\right)|\Delta(z)|^{2},
$$

the normalization by the unitary group volume $V_{U(N)}$ being hidden into the definition of integration measure in (1.1). The last integral has a natural interpretation in terms of the partition function of Coulomb gas of particles with coordinates $z_{i}$, confined by the potential in the exponent.

Almost the same eigenvalue integral can be presented as the partition function of the model of two hermitian non-commuting matrices $X, Y$ (H2MM)

$$
\mathcal{Z}_{N}^{(H 2 M M)}[t, \tilde{t}]=\int \mathcal{D} X \mathcal{D} Y e^{-\operatorname{Tr} X Y+\operatorname{Tr} W(X)+\operatorname{Tr} \tilde{W}(Y)}
$$

2 which is a particular case of the models defined in 23

3 after taking into account the Jacobian of the angular part of commuting matrices 
where $W(X)=\sum_{k=1}^{K} t_{k} X^{k}$ and $\tilde{W}(Y)=\sum_{k=1}^{\tilde{K}} \tilde{t}_{k} Y^{k}$ or, in terms of eigenvalues (using, as usual, the Harish-Chandra-Itzykson-Zuber (HCIZ) formula):

$$
\mathcal{Z}_{N}^{(H 2 M M)}[t, \tilde{t}]=\int \prod_{m=1}^{N}\left(d x_{m} d y_{m} e^{-x_{m} y_{m}+W\left(x_{m}\right)+\tilde{W}\left(y_{m}\right)}\right) \Delta(x) \Delta(y)
$$

Now the Jacobian gives $\Delta(x)^{2} \Delta(y)^{2}$ in contrast to NMM, but the extra powers of Vandermonde determinants are canceled by the HCIZ integral.

Indeed, it is not difficult to notice that the H2MM is very similar to NMM if we take in the former $K=\tilde{K}, \tilde{t}_{k}=\bar{t}_{k}, k=1,2, \ldots$ and compare the eigenvalue representations (1.2) and (1.4). We see that the difference is only in the fact that in (1.2) the eigenvalues of two matrices are complex conjugate, whereas in (1.3) they form two independent real sets. The number of the integration variables is the same, only the contours of integration are different. It suggests that for a large class of potentials $W$ with good convergence properties at infinity (in particular, for many polynomial potentials) the partition functions should be equal, and the correlators of the quantities $\operatorname{Tr} \Phi^{n}, \operatorname{Tr} \Phi^{\dagger m}$ should be the same as correlators of $\operatorname{Tr} X^{n}$, $\operatorname{Tr} Y^{m}$. In what follows we will mostly consider the symmetric case $K=\tilde{K}$ and in particular examples even restrict ourselves to the case of real coefficients $t_{k}=\bar{t}_{k}$.

In the large $N$ limit the models will be also equivalent for most of the potentials $W$. The solution to the saddle point equation for H2MM gives rise in general to two sets of complex conjugated eigenvalues: $x_{m}=z_{m}, y_{m}=\bar{z}_{m} m=1, \ldots, N$, since it is the only way to make the result for the partition function real for a general set of complex couplings $t_{k}$. Hence they will form the same spots of the two dimensional Coulomb charges with the uniform density $\rho(z, \bar{z})=-\partial \bar{\partial} V(z, \bar{z})=1$ as in the case of NMM [24], [9].

The simplest demonstration of this equivalence comes from the direct calculation of the Gaussian H2MM integral

$$
\begin{aligned}
\mathcal{Z}_{N}^{\text {Gauss }} & =\int \mathcal{D} X \mathcal{D} Y e^{\operatorname{Tr}\left(-X Y+t_{1} X+\bar{t}_{1} Y+t_{2} X^{2}+\bar{t}_{2} Y^{2}\right)} \\
& =\left(\frac{4 \pi}{1-4 t_{2} \bar{t}_{2}}\right)^{N^{2} / 2} \exp N^{2}\left(\frac{\bar{t}_{1} t_{1}+\bar{t}_{1}^{2} t_{2}+t_{1}^{2} \bar{t}_{2}}{1-4 t_{2} \bar{t}_{2}}\right)
\end{aligned}
$$

which of course coincides with the partition function of NMM with the same quadratic potential $W(z)$. The latter has in the large $N$ approximation the distribution of the eigenvalues in the shape of ellipse [24], [25], and the coincidence of results confirms our statement that in the saddle point approximation the eigenvalues $x_{k}, y_{k}$ will be complex conjugate and will also form the same ellipse. Both NMM [9] and H2MM [10] were proposed as matrix models describing the two dimensional Laplacian growth processes.

In the next section we will demonstrate the planar diagram technique for the one and two cut H2MM, relating it to the Ising model and to the two phase Ising model on 
planar graphs, respectively. In section 3 we will reproduce (by an unusual method) the solution of the two matrix model in the planar approximation. Using this solution we will build in section 4 the general algebraic curve (in general not hyperelliptic) describing the multi-support two matrix model. We will describe the topology of its Riemann surface and its possible degenerations into lower genera. In section 5 the free energy of the model will be presented as a tau-function in terms of the variables corresponding to the periods of holomorphic differentials of the curve. The cubic case with the two cut degeneration will be considered in detail and a rather explicit solution for its free energy will be given. In section 6 we will describe the connection of the two matrix models (and of some of their generalizations) to the calculation of effective superpotentials of $\mathcal{N}=1$ super Yang-Mills theory with two adjoint chiral multiplets and an appropriate tree superpotential, in relation to the conjecture of [19]. In section 7 we will sketch out the construction of the algebraic curves for a very general class of solvable matrix models with the tree-like interactions of the matrices.

\section{Combinatorics of planar graphs of the multi-support two-matrix model}

The equivalence of NMM and H2MM is useful to give a combinatorial interpretation to the NMM in terms of the planar graph counting. The NMM does not have such a direct interpretation but the H2MM does have it. Indeed, the equivalence between (1.1) and (1.3) suggests the following recipe: if we want to calculate the partition function or the correla-

tors of traces of normal matrices (without mixture of two matrices inside each trace!) we simply have to calculate the corresponding quantities in the corresponding H2MM, taking arbitrary hermitian matrices $X, Y$ instead of the commuting complex matrices $\Phi, \Phi^{\dagger}$, and the same set of complex conjugate couplings $t_{n}, \bar{t}_{n}$. We can do it by all available methods in the H2MM: saddle point approximation, orthogonal polynomials or loop equations, or even perturbatively in the couplings, by the direct planar graph expansion.

\subsection{One-support case: Ising spins on planar Feynman graphs}

Let us remind that the H2MM was used in [3] to define and solve exactly the Ising model on dynamical planar lattices. The role of these lattices is played by planar Feynman graphs and the positions of each spin correspond to two types of interaction vertices $(X$ vertex and $Y$-vertex as spin-up and spin-down). In this respect, we can say that the NMM also describes the Ising model on planar graphs. The phases of the complex couplings correspond here to some generalized (imaginary) magnetic fields, whose values depend on the phases of couplings. 
For example the two matrix model with the cubic interactions describes the statistics of Ising spins on $\Phi^{3}$-type planar graphs (or, due to the Kramers-Wannier duality, on planar triangulations):

$$
\mathcal{Z}_{N}^{(I)}[\gamma, \lambda, \bar{\lambda}]=\int \mathcal{D} \Phi \mathcal{D} \Phi^{\dagger} e^{\operatorname{Tr}\left(-\Phi^{\dagger} \Phi+\frac{\gamma}{2}\left(\Phi^{2}+\Phi^{\dagger 2}\right)+\frac{\lambda}{3} \Phi^{3}+\frac{\bar{\lambda}}{3} \Phi^{\dagger 3}\right)}
$$

It corresponds to the following choice of couplings in (1.1): $t_{1}=\bar{t}_{1}=0, t_{2}=\bar{t}_{2}=\gamma / 2$, $t_{3}=\lambda / 3, \bar{t}_{3}=\bar{\lambda} / 3$. Note that this choice of couplings does not lead to the loss of generality: any cubic potential can be brought to that used in (2.1) by constant shifts of matrices $X \rightarrow X+$ const, $Y \rightarrow Y+\overline{\text { const }}$ and the phase rotations $X \rightarrow e^{i \theta} X, Y \rightarrow e^{-i \theta} Y$.

Let us denote $\lambda=g e^{i H}$. The planar $\Phi^{3}$-graphs are generated in the large $N$ limit by the expansion of (2.1) in powers of $g$, and the corresponding Feynman rules can be given the statistical mechanical interpretation in terms of the Ising model on $\Phi^{3}$-type planar graphs with the temperature $\frac{2}{\log \gamma}$ and the imaginary constant magnetic field $i H$ [3].

The solution of two matrix model describing the Ising spins on planar graphs corresponds to the situation when the eigenvalues for both matrices form one connected support around the classical minimum of the potential corresponding to $\Phi=\Phi^{\dagger}=0$.

\subsection{Multi-support case: multi-Ising phases}

Both NMM and H2MM admit in the large $N$ limit the multi-support solutions, in analogy with the hermitian one matrix model, where they were studied from the point of view of their relation to the hyperelliptic curve in the works [17], [26], [18], and recently in [19] and [20]. In the case of NMM the eigenvalues $z_{k}, \bar{z}_{k}$ are distributed with the constant density in a set of disconnected spots on the complex plane $z$. Our main purpose in this paper is to describe and classify such solutions from the point of view of the underlying algebraic curves.

We will work in the notations corresponding to the complex conjugated $z_{k}, \bar{z}_{k}$ of the NMM. However, all results will be true for the independent $z_{k}, \bar{z}_{k}$, as in the H2MM.

The eigenvalue supports appear around the extrema of the potentials. Note that in the sense of analytic continuation one can also formally "fill up" all extrema of the potential and not only the minima. This leads to more general solutions [19] leading to important physical applications. The extrema of the potential $V(z, \bar{z})=-z \bar{z}+W(z)+\bar{W}(\bar{z})$ are at the points defined by the system of equations

$$
\bar{z}=W^{\prime}(z), \quad z=\bar{W}^{\prime}(\bar{z})
$$

In general, for the potentials of degree $(n+1)$ we have $n^{2}$ extrema. 
To be more concrete let us study the case of cubic polynomial potential (related to the one in (2.1) by a simple shift of variables):

$$
V(z, \bar{z})=-z \bar{z}+T(z+\bar{z})+\frac{g}{3}\left(z^{3}+\bar{z}^{3}\right)
$$

with real couplings $T$ and $g$, and fill out only the two extrema obeying the reality condition $z=\bar{z}$. The classical equations (2.2) for the extrema of (2.3) can be rewritten in the form of a "classical" curve 4

$$
\begin{array}{r}
\frac{1}{g^{2}}\left(\bar{z}-g z^{2}-T\right)\left(z-g \bar{z}^{2}-T\right)= \\
=z^{2} \bar{z}^{2}-\frac{1}{g}\left(z^{3}+\bar{z}^{3}\right)+\frac{T}{g}\left(z^{2}+\bar{z}^{2}\right)+\frac{1}{g^{2}} z \bar{z}-\frac{T}{g^{2}}(z+\bar{z})+\frac{T^{2}}{g^{2}}=0
\end{array}
$$

and the solution has $n^{2}=4$ extrema, two extrema for $z=\bar{z}$ and another two for $z+\bar{z}=$ $-1 / g$. The potential can be expanded around the extrema $z=\bar{z}$ as follows

$$
\begin{array}{r}
V(z, \bar{z})=-\left(z-\hat{z}_{a}\right)\left(\bar{z}-\hat{z}_{a}\right)+\frac{m_{a}}{2}\left(\left(z-\hat{z}_{a}\right)^{2}+\left(\bar{z}-\hat{z}_{a}\right)^{2}\right)+ \\
+\frac{g}{3}\left(\left(z-\hat{z}_{a}\right)^{3}+\left(\bar{z}-\hat{z}_{a}\right)^{3}\right) \pm \mathrm{const}
\end{array}
$$

where $a=1,2$,

$$
\hat{z}_{1,2}=\frac{1}{2 g}(1 \pm \sqrt{1-4 T g})
$$

and $m_{1,2}=2 g \hat{z}_{1,2}$. We will not consider the filling of the spots corresponding to other extrema, with $z+\bar{z}=-1 / g$ (later we will discuss this fact in a more general context).

Let us regroup the eigenvalues into two groups and denote:

$$
\begin{aligned}
\left(z_{1}-\hat{z}_{1}, \ldots, z_{N_{1}}-\hat{z}_{1}\right) & =\left(a_{1}, \ldots, a_{N_{1}}\right) \\
\left(z_{N_{1}+1}-\hat{z}_{2}, \ldots, z_{N}-\hat{z}_{2}\right) & \equiv\left(b_{1}, \ldots, b_{N_{2}}\right), \quad N_{1}+N_{2}=N
\end{aligned}
$$

and the same for the conjugated variables 0 , corresponding to their positions in the first or second spot, respectively. Now we can use the eigenvalue representation (1.2) and rewrite this integral in terms of hermitian matrices $A, \tilde{A}$, having the size $N_{1} \times N_{1}$ and $B, \tilde{B}$ having the size $N_{2} \times N_{2}$ and a pair of complex rectangular anticommuting ghost matrices $C, \tilde{C}$ having the size $N_{1} \times N_{2}$, as follows:

$$
\mathcal{Z}_{N}[t, \tilde{t}]=\int \mathcal{D} A \mathcal{D} \tilde{A} \mathcal{D} B \mathcal{D} \tilde{B} \mathcal{D} C \mathcal{D} \tilde{C} e^{N \operatorname{Tr} S(A, \tilde{A}, B, \tilde{B}, C, \tilde{C})}
$$

\footnotetext{
4 Compare it to the curve for the one-support solution in 27]

5 In the case of the H2MM one can imagine the situation when the filling numbers of the variables $\bar{z}_{i}$ are not the same as for $z_{i}$ 's: $N_{1}^{\prime} \neq N_{1}, N_{2}^{\prime} \neq N_{2}$; we don't consider this situation here.
} 


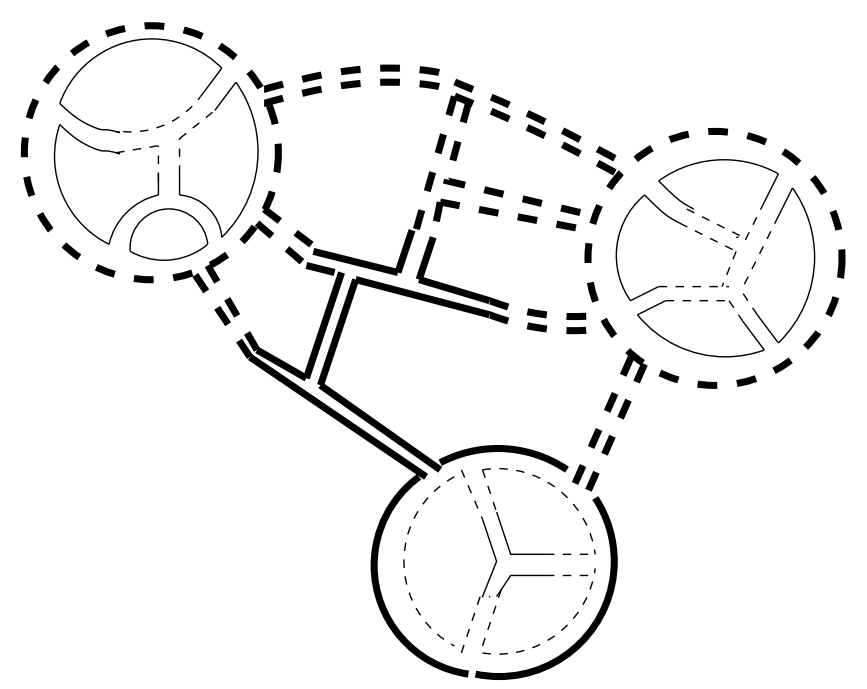

Fig. 1: A planar graph of the two matrix model with two eigenvalue supports. There are two phases here: thin line phase (inside the circles) and thick line phase (outside the circles). Each phase corresponds to two different kinds of Ising spins having a different temperature: the spins looking "up" are located in the triple vertices made of solid double lines, and the spins looking "down" are located in the triple vertices made of dotted double lines. The three types of propagators inside each phase (solid, dotted and mixed) describe the interactions depending on the mutual orientation of the neighboring spins. Along the interphase (ghost) lines drown by circles, the spins have the same orientation.

where

$$
\begin{aligned}
S(A, \tilde{A}, B, \tilde{B}, C, \tilde{C})= & -A \tilde{A}+\frac{m_{1}}{2}\left(A^{2}+\tilde{A}^{2}\right)+\frac{g}{3}\left(A^{3}+\tilde{A}^{3}\right) \\
& -B \tilde{B}+\frac{m_{2}}{2}\left(B^{2}+\tilde{B}^{2}\right)+\frac{g}{3}\left(B^{3}+\tilde{B}^{3}\right) \\
& -m C^{\dagger} C-m \tilde{C}^{\dagger} \tilde{C}-g C^{\dagger} C A+g C C^{\dagger} B-g \tilde{C} \tilde{C}^{\dagger} \tilde{A}+g \tilde{C} \tilde{C}^{\dagger} \tilde{B}
\end{aligned}
$$

and $m=\sqrt{1-4 T g}$. To rewrite the eigenvalue integrals as the matrix ones, we used the HCIZ formula for the $U\left(N_{1,2}\right)$ group integral

$$
\int[d \Omega]_{U\left(N_{1}\right)} e^{\operatorname{Tr}\left(\Omega^{\dagger} x \Omega \bar{x}\right)} \propto \frac{\operatorname{det}_{i j} e^{a_{i} \bar{a}_{j}}}{\Delta(a) \Delta(\bar{a})}
$$

and similarly for $b, \bar{b}$. The matrices $C, \tilde{C}$ served to exponentiate the cross-products $\prod_{k, m}\left(a_{k}-b_{m}\right)\left(\bar{a}_{k}-\bar{b}_{m}\right)$ in the Vandermonde determinants in (1.2) (see the similar method for the one matrix model in [17], [20]).

Now we can give the model (2.9) a combinatorial interpretation in terms of the planar graph expansion. Namely, we expand (2.8) in the cubic coupling $g$, for the fixed $m_{1}, m_{2}, m$. 
The diagram technique consists of the following elements:

$$
\text { propagators : } \begin{aligned}
& \langle A A\rangle_{0}=\langle\tilde{A} \tilde{A}\rangle_{0}=\frac{2 m_{1}}{m_{1}^{2}-1},\langle A \tilde{A}\rangle_{0}=\frac{2}{m_{1}^{2}-1} ; \\
& \langle B B\rangle_{0}=\langle\tilde{B} \tilde{B}\rangle_{0}=\frac{2 m_{2}}{m_{2}^{2}-1},\langle B \tilde{B}\rangle_{0}=\frac{2}{m_{2}^{2}-1} \\
& \left\langle C^{\dagger} C\right\rangle_{0}=\left\langle\tilde{C} \tilde{C}^{\dagger} \tilde{C}\right\rangle_{0}=1 / m
\end{aligned}
$$

Each type of the propagators $\left\langle C^{\dagger} C\right\rangle_{0}$ and $\left\langle\tilde{C}^{\dagger} \tilde{C}\right\rangle_{0}$ forms closed loops on Feynman graphs, each loop entering with the factor $(-1)$.

A typical planar graph for the two-support model is presented on fig. 1. Let us classify the index loops of each planar graph (or a graph of a fixed topology) as carrying the index $i=1, \ldots, N_{1}$ (solid line), or the index $i^{\prime}=1, \ldots, N_{2}$ (dotted line). The ghost loops (drawn by a double line formed by a thick and a thin line) will separate two phases on the planar graph: one described by the matrices $A, \tilde{A}$ (thick line phase) and another described by the matrices $B, \tilde{B}$ (thin line phase). Each of these phases corresponds to the dynamics Ising spins of 1-st and 2-nd kind, as described in the previous subsection for the single support case. At the phase boundaries formed by the ghost loops, the spins have the same orientation (two types of ghosts $C$ and $\tilde{C}$ correspond to two possible orientations). Each solid index loop contributes a factor $N_{1}$, and each dotted index loop - a factor $N_{2}$.

Let us note at this point that for $g T<1 / 4$ we always have real $m_{1}>0, m_{2}>0$, but the determinants of second derivatives of the action at two different extrema are $m_{1}^{2}-1>0$ and $m_{2}^{2}-1<0$ correspondingly, which means that the first extremum is the true minimum, and the second is a saddle point of the potential.

The last comment about this diagram technique: as it was done in [20] for the twocut one matrix model, we can do the following formal operation with each graph, without changing its contribution: we can change the contribution of each ghost loop from $(-1)$ to 1, change the sign of each $B B, \tilde{B} \tilde{B}$ and $B \tilde{B}$ propagator, and the sign of $N_{2}$.

All this means that we can consider instead of the matrix model (2.8)-(2.9), the matrix model with the action:

$$
\begin{aligned}
S(A, \tilde{A}, B, \tilde{B}, C, \tilde{C})= & -A \tilde{A}+\frac{m_{1}}{2}\left(A^{2}+\tilde{A}^{2}\right)+\frac{g}{3}\left(A^{3}+\tilde{A}^{3}\right) \\
& +B \tilde{B}-\frac{m_{2}}{2}\left(B^{2}+\tilde{B}^{2}\right)+\frac{g}{3}\left(B^{3}+\tilde{B}^{3}\right) \\
& -m C^{\dagger} C-m \tilde{C}^{\dagger} \tilde{C}+g C^{\dagger} C A+g C C^{\dagger} B+g \tilde{C} \tilde{C}^{\dagger} \tilde{A}+g \tilde{C} \tilde{C}^{\dagger} \tilde{B}
\end{aligned}
$$

6 we will see in the next sections that $g N_{1} / N$ and $g N_{2} / N$ can be viewed as independent variables in the planar limit, as in the one matrix model case [20] 
Here $C$ and $\tilde{C}$ are already the usual commuting complex $N_{1} \times N_{2}$ rectangular matrices (the sign of $N_{2}$ is again normal here). We also changed the variables as follows $A \rightarrow-A$, $\tilde{A} \rightarrow-\tilde{A}$.

In this new representation of the same model, the perturbative $g$-expansion goes around the true minima of the potential, and the contributions of planar graphs are positive. The planar expansion of this matrix model define the statistical mechanical model on random dynamical graphs describing a two phase system, each phase corresponding to the system of Ising spins with the ferromagnetic boundary condition on the phase boundary. Note that since we have two independent "cosmological constants": the coupling $g$ and $N_{2} / N$, but only one parameter $T$ related to the (different) temperatures of two kinds of Ising spins. Hence we cannot make both types of the Ising spins critical at the same time. We need for that higher powers of the potential. In a sense, our multi-cut solution generalizes the ADE models proposed in [28], 229].

Let us conclude this section by noticing that much of what we did here on the two support case can be carried over to the 4-support case of this model and to the multiple supports for the potentials of higher degree. However, unlike the cubic case with real couplings, the details are difficult to work out. Below we will return to the cubic potential and discuss in detail the generic 4-support structure. We will also see that the generic 4-support solution has a very natural 2-support "degeneration", corresponding precisely to the perturbation theory considered in this section.

\section{Solution of the model in the planar limit}

Let us now turn to the solution of the two matrix model in the planar or large $N$ limit. As is well-known in this case the computation of matrix integrals (1.1) or (1.3) can be reduced to the solution of the saddle point equation.

The saddle point equation for the model (1.2) with the eigenvalues $z_{1}, \ldots, z_{N}$ (or analytically continued saddle point equation for (1.4)) reads

$$
\bar{z}_{k}=W^{\prime}\left(z_{k}\right)+\sum_{j(\neq k)} \frac{1}{z_{k}-z_{j}}
$$

together with the complex conjugated equation. For the resolvents of distributions of the eigenvalues

$$
G(z)=\hbar\left\langle\operatorname{Tr} \frac{1}{z-\Phi}\right\rangle, \quad \bar{G}(\bar{z})=\hbar\left\langle\operatorname{Tr} \frac{1}{\bar{z}-\Phi^{\dagger}}\right\rangle
$$

it can be written as:

$$
\begin{aligned}
& \bar{z}=W^{\prime}(z)+G(z) \\
& z=\bar{W}^{\prime}(\bar{z})+\bar{G}(\bar{z})
\end{aligned}
$$


where the resolvent has the usual asymptotics at large $z$ or $\bar{z}$ for the finite supports:

$$
G(z) \rightarrow t_{0} / z+O\left(1 / z^{2}\right), \quad \bar{G}(\bar{z}) \rightarrow t_{0} / \bar{z}+O\left(1 / \bar{z}^{2}\right)
$$

To fix the resolvents in (3.3)we have to impose the condition that the functions $\bar{z}(z)$ and $z(\bar{z})$ are mutually inverse:

$$
\bar{z}(z(x))=x
$$

To justify this condition we recall once again that the solutions of these equations describe the spots of Coulomb charges with the uniform distribution of the eigenvalues with coordinates $\left(z_{1}, \bar{z}_{1}\right), \ldots,\left(z_{N}, \bar{z}_{N}\right)$ with the density $\rho(z, \bar{z})=1$. The boundaries of the spots are in general smooth curves in the complex plane $z$ depending on the couplings of the potential. To fix the form of these boundaries it is enough to consider the eqs. (3.3) at the boundary. Then both equations should define the same curve $\bar{z}(z)$. It means that the solutions of these equations, $\bar{z}(z)$ and $z(\bar{z})$ respectively, should be mutually inverse, i.e. obey eq. (3.5) the complex manifold (with involution) and it becomes literally complex conjugated to the function $z$ only on some real section - the real analytic curve in the sense of [9], which is just a boundary of the eigenvalue distribution. To avoid further misunderstanding in what follows we will denote this function as $\tilde{z}(z)$, so that $\tilde{z}(z)=\bar{z}$ (i.e. is literally complex conjugated only on the boundaries of the spots).

In the quasiclassical, or dispersionless limit one considers the free energy of the matrix ensembles to be defined as a "planar" limit

$$
\mathcal{F}(t, S)=\lim \left(\hbar^{2} \log \mathcal{Z}\left(\frac{t}{\hbar}\right)\right)
$$

implying $N \rightarrow \infty, \hbar \rightarrow 0$ with $N \hbar=t_{0}$ being fixed. In (3.6) $t$ denote the parameters of the potential $V(z, \bar{z})=-z \bar{z}+W(z)+\bar{W}(\bar{z})$ while $S$ are the new variables directly related to the "filling numbers" of various eigenvalue supports. More strictly, by the planar limit (3.6) one usually understands the solution to the variational problem

$$
\begin{array}{r}
\mathcal{F} \propto \int V(z, \bar{z}) \rho(z, \bar{z}) d^{2} z-\int d^{2} z_{1} d^{2} z_{2} \rho\left(z_{1}, \bar{z}_{1}\right) \rho\left(z_{2}, \bar{z}_{2}\right) \log \left|z_{1}-z_{2}\right|+ \\
+\sum_{\alpha} v_{\alpha}\left(\int \rho(z, \bar{z}) d^{2} z-S_{\alpha}\right)
\end{array}
$$

7 A more rigorous derivation of these equations from the method of orthogonal polynomials can be found in [5], [6]. 
which is the "stationary phase" condition for corresponding matrix integrals (1.2), (1.4). Note that the normalization of the density at different supports is achieved by the Langange multipliers $v_{\alpha}$. The dispersionless tau-function can be obtained from (3.7) by the substitution of the saddle point solution $\rho=\rho_{c}$ of the saddle point equation

or

$$
\frac{\delta \mathcal{F}}{\delta \rho(z, \bar{z})}=0
$$

$$
v_{\alpha}=\int d^{2} z^{\prime} \rho\left(z^{\prime}, \bar{z}^{\prime}\right) \log \left|z_{\alpha}-z^{\prime}\right|-V\left(z_{\alpha}, \bar{z}_{\alpha}\right)
$$

for any point $P_{\alpha}=\left(z_{\alpha}, \bar{z}_{\alpha}\right)$ belonging to one of the supports, labelled by $\alpha$.

In the next two sections we will first discuss the structure of the complex curve (3.3) for two matrix model and then define the free energy (3.7) as a (logarithm) of quasiclassical tau-function.

\section{Complex curve for the two-matrix model}

The reality condition suggests the following ansatz for the solution to (3.3):

$$
F(z, \tilde{z})=\sum_{i, j} f_{i j} z^{i} \tilde{z}^{j}=0
$$

with the coefficients obeying the symmetry: $f_{i j}=\bar{f}_{j i}$ 目. Due to this symmetry the equation (3.5) will be automatically satisfied.

The coefficients $f_{i j}$ can be partially fixed by the asymptotics:

$$
\left.\tilde{z}(z)\right|_{z \rightarrow \infty} \simeq W^{\prime}(z)+\frac{t_{0}}{z}+O\left(\frac{1}{z^{2}}\right)
$$

following from (3.3) and (3.4), but the number of parameters of the potential grows linearly with its degree while the number of coefficients of (4.1) grows quadratically. The rest of the parameters will correspond to the eigenvalue filling numbers for various spots (supports of the eigenvalues on the $z$ plane). Altogether they will play the role of moduli of complex structure of the algebraic curve defined by the eq. (4.1).

One may also think of the analytic curve (4.1) as of the algebraic form of the large $N$ loop equations in H2MM (but not in NMM!) for the resolvent $G(z)$ [30], 27], or for the matrix model in external field [31], where it was first proposed.

\subsection{Structure of the curve}

We can precise the algebraic equation of the curve (4.1) for the mutually complex conjugated potentials of a degree $K=n+1$ (with a few explicitly given highest degree terms)

$$
F(z, \tilde{z})=z^{n} \tilde{z}^{n}+a z^{n+1}+\bar{a} \tilde{z}^{n+1}+\sum_{i, j \in(N . P .)_{+}} f_{i j} z^{i} \tilde{z}^{j}=0
$$

8 since any such curve should be consistent with its real section, the eq. $F(x+i y, x-i y)=$ $\mathcal{P}(x, y)=0$ with real coefficients. 


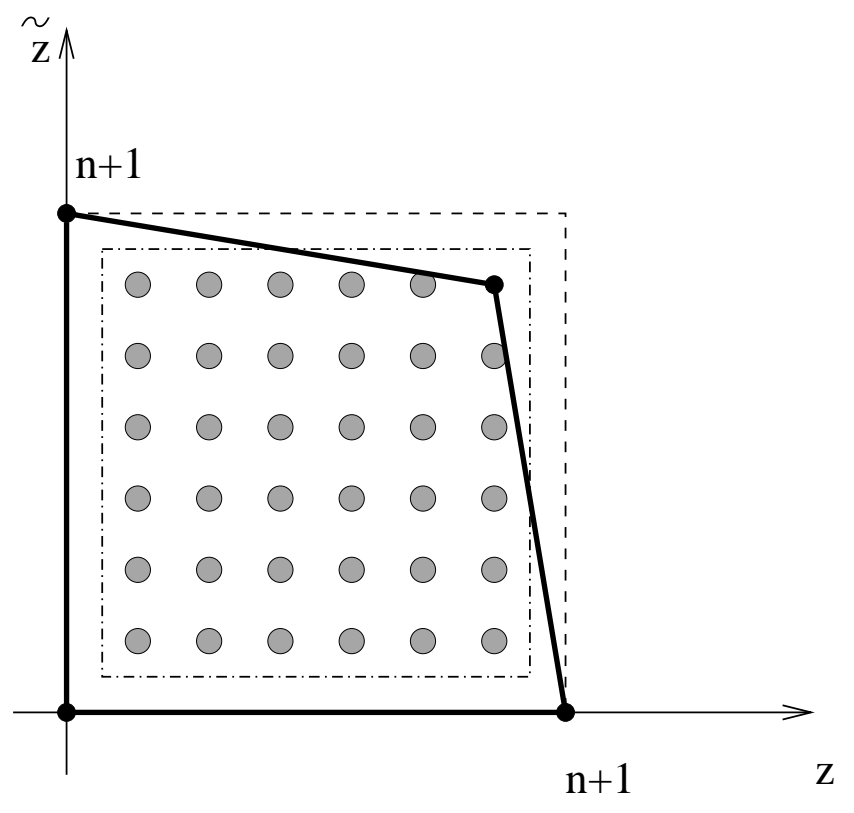

Fig. 2: The Newton polygon for the curve (4.2). The highest degree terms in (4.2) determine the shape of the polygon and the integer dots inside it count the number of holomorphic differentials, or genus of the curve. Clearly this number is equal to the area of "dual" square except for one (black) point, so that $g=n^{2}-1$.

where the first three terms correspond to the three points on the boundary lines of the Newton polygon (square in this case) on fig. 2, and the sum over (N.P.) $)_{+}$in the last term means the sum over the points inside the Newton polygon (including the points on both axis not marked on fig. 2). For example, there are 8 terms in this sum for $n=2$.

One may compare this equation with (2.4) and see that the higher degree terms are always fixed by the "classical" equations on extrema of the matrix model potential. The properties of the curve $((4.2))$ can always be easily established via the Newton polygon on fig. 2 .

Counting the number of integer points inside the polygon one finds that the number of holomorphic differentials, or genus of the curve, is equal to

$$
g=n^{2}-1 .
$$

A simple basis for the holomorphic differentials can be chosen as

$$
d v_{i j}=z^{i} \tilde{z}^{j} \frac{d \tilde{z}}{F_{z}}=-z^{i} \tilde{z}^{j} \frac{d z}{F_{\tilde{z}}},
$$

with the degrees $i=i^{\prime}-1$ and $j=j^{\prime}-1$, where $\left(i^{\prime}, j^{\prime}\right) \in N . P$. are coordinates of the points strictly inside the Newton polygon, without the boundary points (see fig. 2) 9 .

9 For example, for $n=2$ there are three points inside the polygon: $i^{\prime}, j^{\prime}>0$ and $i^{\prime}+j^{\prime} \leq 2$, then the holomorphic differentials are labeled by $i, j \geq 0$ and $i+j \leq 1$. 
Finally let us point out here that for the models with non-symmetric potentials $W$ and $\tilde{W}$ one may write the spectral curve equation in a similar way, but it would not obey such symmetric properties. For the potentials $W$ and $\tilde{W}$ of degrees $n+1$ and $\tilde{n}+1$, correspondingly, the highest terms will always be of a particular form (one may equally use here $x$ and $y$ instead of $z$ and $\tilde{z}$, to show its direct relation to (1.4))

$$
F(z, \tilde{z})=z^{n} \tilde{z}^{\tilde{n}}+A z^{n+1}+B \tilde{z}^{\tilde{n}+1}+\sum_{(i, j) \in(N . P .)_{+}} f_{i j} z^{i} \tilde{z}^{j}
$$

and the genus of the curve (4.5) is $n \tilde{n}-1$. It means that the $(n+1) \times(n+1)$ square on fig. 2 should be replaced by the rectangle of the size $(n+1) \times(\tilde{n}+1)$ with all other elements of the construction remaining intact. Of course, for $\tilde{n}=1$ integrating over the matrix with Gaussian potential one returns to the 1MM with the (hyperelliptic) curve of genus $n-1$ (see section 5.1 below).

\subsection{The cubic example}

To understand better the structure of the curve let us first discuss in detail the cubic example. Writing eq. (4.2) first with arbitrary coefficients

$$
F(z, \tilde{z})=z^{2} \tilde{z}^{2}+a z^{3}+\bar{a} \tilde{z}^{3}+b z^{2} \tilde{z}+\bar{b} z \tilde{z}^{2}+c z^{2}+\bar{c} \tilde{z}^{2}+f z \tilde{z}+q z+\bar{q} \tilde{z}+h=0
$$

one finds that its structure is in fact very similar to the equation of the "classical" curve (2.4). Indeed, the eq. (4.6) should be consistent with the asymptotics

$$
\tilde{z}=W^{\prime}(z)+G(z)=\sum_{k=1}^{3} k t_{k} z^{k-1}+O\left(\frac{1}{z}\right) \equiv \lambda z^{2}+\gamma z+\eta+O\left(\frac{1}{z}\right)
$$

Substituting (4.7) into the eq. (4.6) and collecting the coefficients in front of the terms $z^{6}$, $z^{5}$ and $z^{4}$ one gets

$$
\begin{array}{r}
a=-\frac{1}{\bar{\lambda}}=-\frac{1}{g} \\
b=\frac{\bar{\gamma}}{\bar{\lambda}}=0 \\
c=\frac{\bar{\eta}}{\bar{\lambda}}-\frac{\gamma}{\lambda \bar{\lambda}}+2 \frac{\bar{\gamma}^{2}}{\bar{\lambda}^{2}}=\frac{T}{g}
\end{array}
$$

and their complex conjugated counterparts, i.e. the coefficients at higher degree terms are indeed completely fixed by parameters of the potential (2.3). Four lower degree coefficients $f, q, \bar{q}$ and $h$ correspond to the bipole differential and three holomorphic differentials 10 . Their classical "expectation values" are presented in eq. (2.4).

10 Note, that the curve (4.2), (4.6) is written implying some reality condition onto the coefficients, but as usual, the deformations of these coefficients should be considered as independent complex variables. 


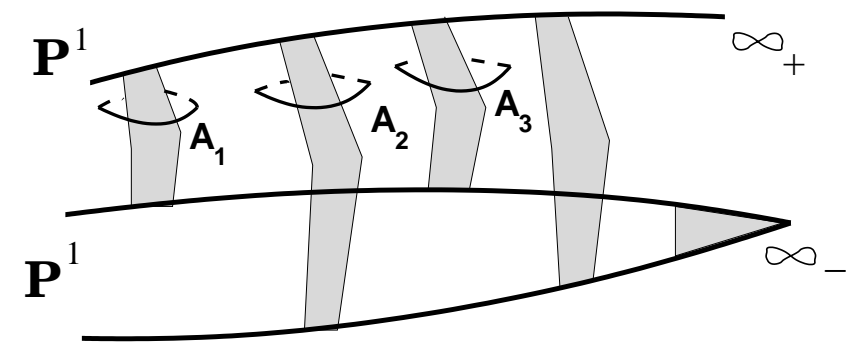

Fig. 3: Cubic curve as a cover of $z$-plane.

Let us now present the curve (4.6) as a Riemann surface of a multi-valued function $\tilde{z}(z)$. Then it can be thought as a three-sheet cover of the complex $z$-plane. On the first, physical sheet, there are no branch cuts at $z \rightarrow \infty$, as it follows from the asymptotics (4.7). This asymptotics should be supplemented by the "complex-conjugated" asymptotics

$$
z=\bar{W}^{\prime}(\tilde{z})+O\left(\frac{1}{\tilde{z}}\right)
$$

on "unphysical" sheets. Then it is clear from (4.7) and (4.9) that on the physical sheet at infinity $\tilde{z} \propto z^{2}$, while on two unphysical sheets $\tilde{z} \propto \sqrt{z}$ and two infinities on unphysical sheets are "glued" by a cut.

The branch points at $z$-plane are determined by zeroes of the differential $d z$, or by $F_{\tilde{z}}=0$. Considering the simplest non-degenerate case of the curve (4.6)

$$
z^{2} \tilde{z}^{2}+a z^{3}+\bar{a} \tilde{z}^{3}+h=0
$$

it is easy to see that there are nine branch points in the $z$-plane without infinity $z=\infty$ (of course, one comes to the same conclusion looking at the Cardano formula, or from the index theorem, see below).

The structure of the curve can be then presented as on fig. 3. It is clear from this picture that the curve can be presented as two copies of $\mathbf{P}^{1}$ "glued" by four cuts, i.e. in general position it has the genus $g=3$. There are two "infinities" $z=\infty, \tilde{z}=\infty$, one of them is a branch point. We have shown schematically the possible cuts and the corresponding choice of canonical A-cycles. In the classical situation (2.4) one has two parabolas intersecting at four points, and under quantum resolution these points turn into four cuts connecting two $\mathbf{P}^{1}$ 's in fig. 3 . 


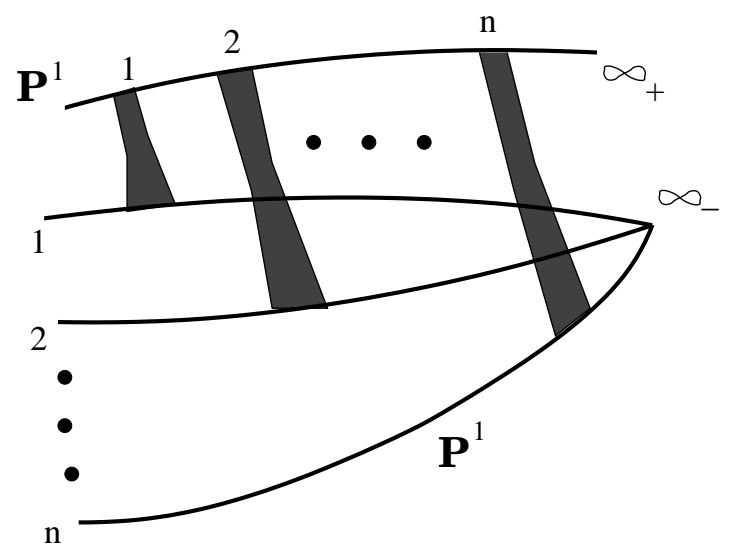

Fig. 4: Generic curve of the two matrix model as a cover of the $z$-plane. In contrast to fig. 3 each fat line consists of a stack of $n$ cuts.

To conclude the picture, let us make few comments about the curve (4.2) for a potential of a generic degree $n$, i.e. when $W^{\prime}(z) \sim z^{n}+\ldots$ This curve (see fig. 4) can be again presented as two $\mathbf{P}^{1}$ 's glued by $n$ stacks of cuts. One of these $\mathbf{P}^{1}$ 's corresponds to the "physical sheet", the other one is glued at the $\infty_{-}$from $n$ copies of "unphysical" $z$-sheets. Each stack consists of $n$ cuts, so their total number is $n^{2}$ among which one can choose $n^{2}-1$ independent, whose number is equal to the genus of this Riemann surface.

The differential $d z$ has always a pole of the second order at $\infty_{+}$on the upper, or "physical" sheet, and a pole of the order $n+1$ at $\infty_{-}$since $\left.z\right|_{\infty_{-}} \propto \tilde{z}^{n}+\ldots$. It gives altogether $n+3$ poles and from the Riemann-Roch theorem one concludes that the number of branching points, or zeroes of $d z$ is equal to

$$
\#(d z=0)=n+3+2\left(n^{2}-1\right)-2=2 n^{2}+n-1
$$

reproducing nine for $n=2$. In general position this gives exactly $2 n^{2}$ branch points, producing simple cuts and $(n-1)$ ramification points, connected by cuts with $\infty_{-}$.

Finally, let us point out that the structure of the curve (4.2) and fig. 4 is consistent with the structure of the "double" in $z$ and $\bar{z}$ variables, explicitly seen in the one-support solution [9] and proposed to be the feature of the multi-support solutions by Krichever [32]. Indeed, the eq. (4.2) is "symmetric" with respect to $z$ and $\tilde{z}$ variables, so instead of the picture on fig. 4, one can draw a "dual" picture of a $(n+1)$-sheet cover of the $\tilde{z}$-plane. These dual pictures can be combined together as on fig. 5. Cutting "physical" sheets from both pictures one may glue them together, forming a double with involution $z \leftrightarrow \bar{z}$. The only delicate point is that these $z$ and $\bar{z}$ sheets should be glued together along the boundaries of the spots where $\tilde{z}(z)=\bar{z}$ and vice versa, in contrast to the picture of fig. 4, where the sheets are glued along the cuts on Riemann surface of the multi valued function $\tilde{z}(z)$ defined by solution to eq. (4.2). Both sheets of the double (the lower picture at fig. 5) generally have $n^{2}$ spots. 

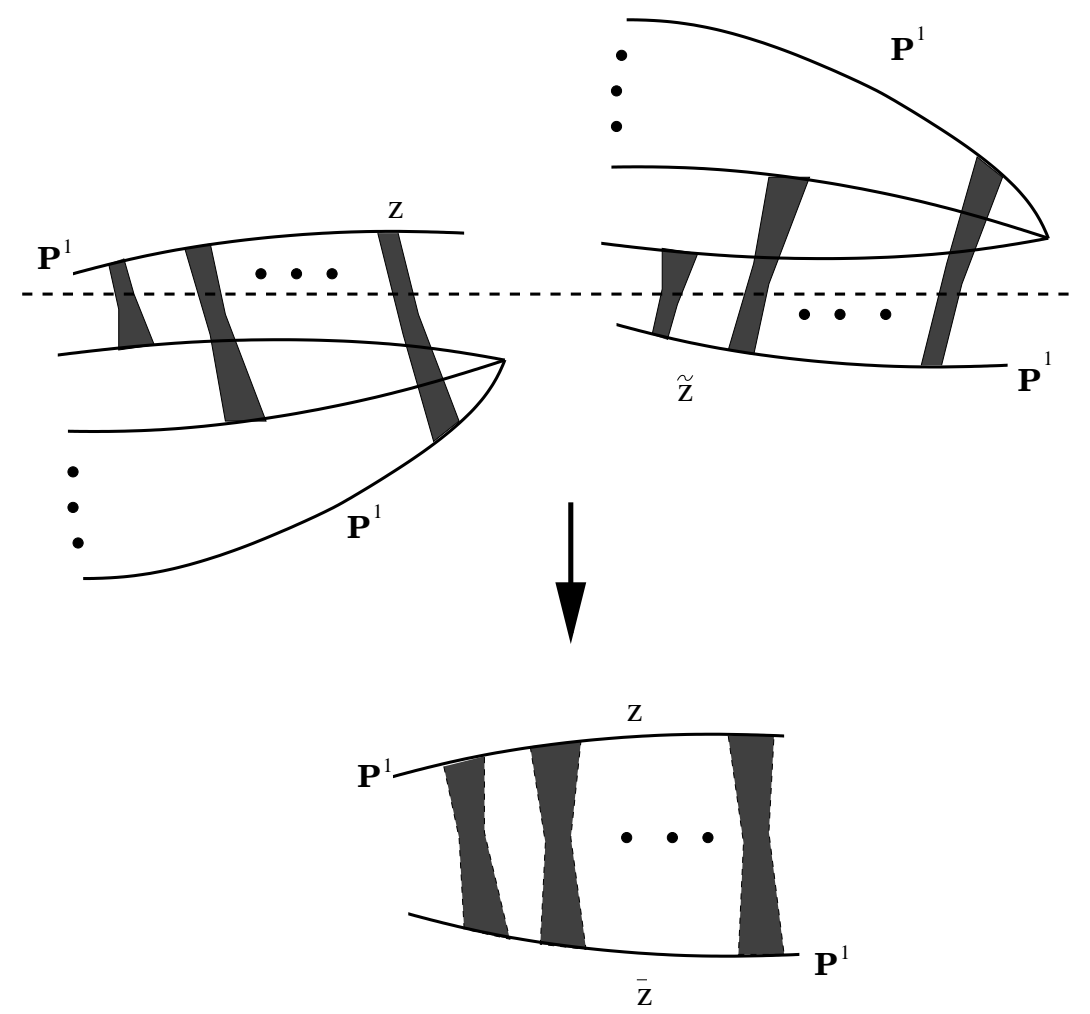

Fig. 5: Generic curve of the two matrix model as a double of $z$ and $\bar{z}$ planes. One takes the Riemann surface of the function $\tilde{z}(z)$, as on fig. 4, and its "mirror" Riemann surface of the function $z(\tilde{z})$ which possesses the same structure. Cutting the physical sheets one may glue them together along the (real) curves $\bar{z}=\tilde{z}(z)$.

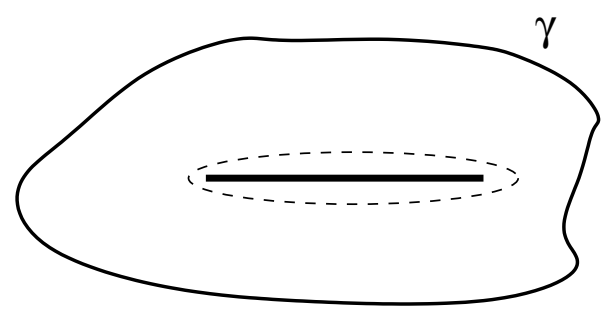

Fig. 6: The boundary of the spot $\gamma$ and a cut of a multi-valued function $\tilde{z}(z)$ inside the spot. On $\gamma$ one has an equality $\bar{z}=\tilde{z}(z)$ but this is not true on the cut.

The difference between the boundary of a spot and a cut of a multi-valued function is demonstrated on fig. 6. One obviously gets the following relations for the two-dimensional and contour integrals

$$
\int_{\text {spot }} d z \wedge d \bar{z}=\oint_{\gamma} \bar{z} d z=\oint_{\gamma} \tilde{z} d z=\oint_{\text {cut }} \tilde{z} d z
$$




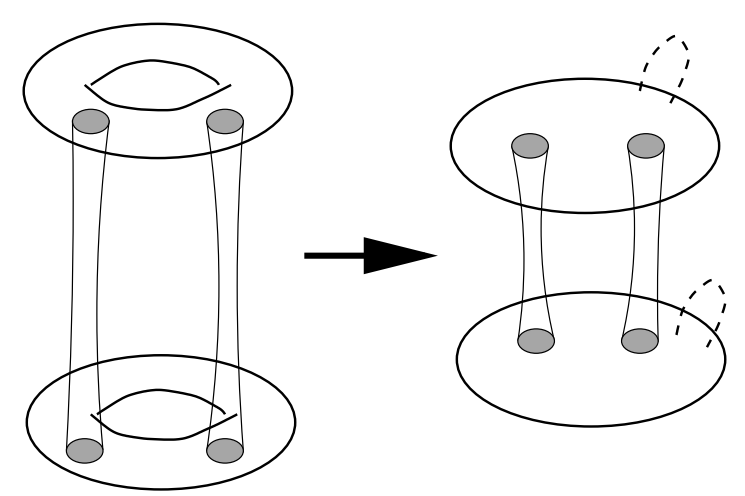

Fig. 7: The curve (4.6) as double cover of the torus. When the torus (4.14) degenerates, the genus $g=n^{2}-1=3$ curve (4.6) degenerates into the curve of $g_{\text {red }}=n-1=1$.

which clarify the equivalence of two pictures on fig. 5. The relations (4.12) allow to endow the complex curve (4.2) (or (4.5) in the asymmetric case) with a meromorphic generating differential $\tilde{z} d z$.

\subsection{Degenerate curves}

Let us now discuss how the curve (4.2) can be degenerated. The (smooth) genus $g=n^{2}-1$ of the curve (4.2) decreases if there exists nontrivial solution to the system of equations

$$
F(z, \tilde{z})=0, \quad d F=0
$$

It imposes certain constraints to the coefficients of $f_{i j}$ of the equation (4.2), which can be found, say computing the resultant of the equations (4.13). However, these constraints cannot be really resolved in a general position.

To get an idea how the curve (4.2) can be degenerated consider first the cubic case (4.6) and let us put all coefficients of this equation to be real. Then, it is easy to see that it can be rewritten in the form

$$
Y^{2}+a X^{3}+c X^{2}+q X+h-\frac{1}{4}((3 a-b) X+2 c-f)^{2} \equiv Y^{2}+P(X)=0 .
$$

where

$$
X=z+\tilde{z}, \quad Y=z \tilde{z}-\frac{1}{2}((3 a-b) X+2 c-f)
$$

One may "tune" for simplicity the coefficients of the potential (4.8) to get $3 a=b$ and $2 c=f$. The formulas (4.15) show that our curve (4.2) can be presented as a double cover of the torus (4.14) with four branch points which are solutions to the equation (4.6) under 


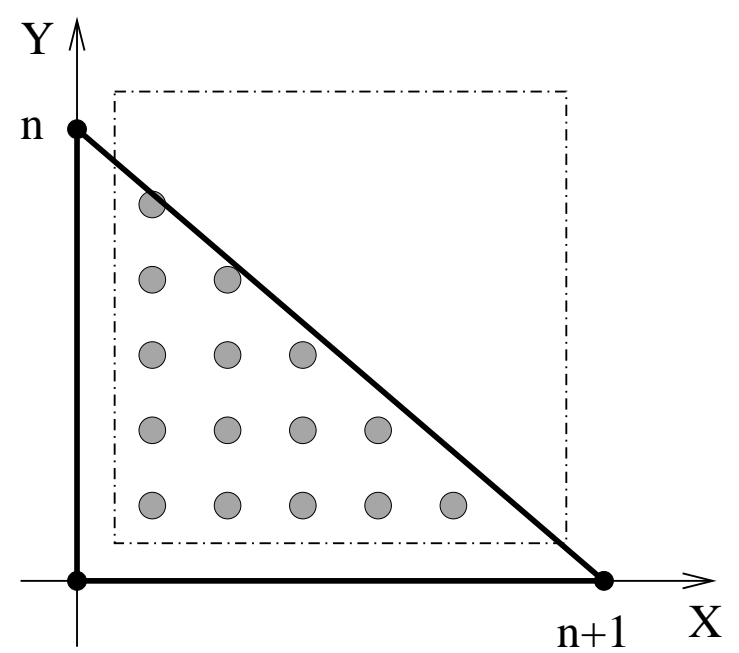

Fig. 8: The Newton polygon for the curve (4.17) gives the genus $g_{*}=\frac{n(n-1)}{2}$.

the substitution $\tilde{z}=z$, where the transformation (4.15) becomes singular. Hence, the curve (4.6) can be also presented (in addition to fig. 3) as two tori glued by two cuts (see fig. 7).

Now it becomes clear how this picture can be degenerated. Rewriting equations (4.13) as

$$
\begin{aligned}
& F_{\tilde{z}}=z F_{Y}+P^{\prime}(X) \\
& F_{\tilde{z}}=\tilde{z} F_{Y}+P^{\prime}(X)
\end{aligned}
$$

one immediately finds that they lead either to $z=\tilde{z}$ or to $F_{Y}=0$ and $P^{\prime}(X)=0$. In the second case the torus (4.14) degenerates, while $z=\tilde{z}$ leads to degeneration of the cover of this torus. We will be more interested in the degeneration of the torus since, for example, it corresponds to filling of the "correct" vacua (real eigenvalues) in the perturbative picture considered in section 2 .

When the torus degenerates into a rational curve, one gets the Riemann surface (4.6) presented as a double cover of this rational curve with two cuts, i.e. as a Riemann surface of genus $g=1$ with smooth handles of tori degenerated into (a pair of) singular points (see fig. 7).

The equations of degeneration of the torus (4.14) can be easily written using the conditions for the double root of the polynomial $P(X)$. Explicitly these conditions acquire the form of the discriminant of $P(X)$ or the resultant of the two polynomials $P(X)$ and $P^{\prime}(X)$.

Now, in the general case (4.2) with real coefficients the substitution analogous to (4.15) brings it to the form

$$
Y^{n}+X^{n+1}+\ldots=0
$$



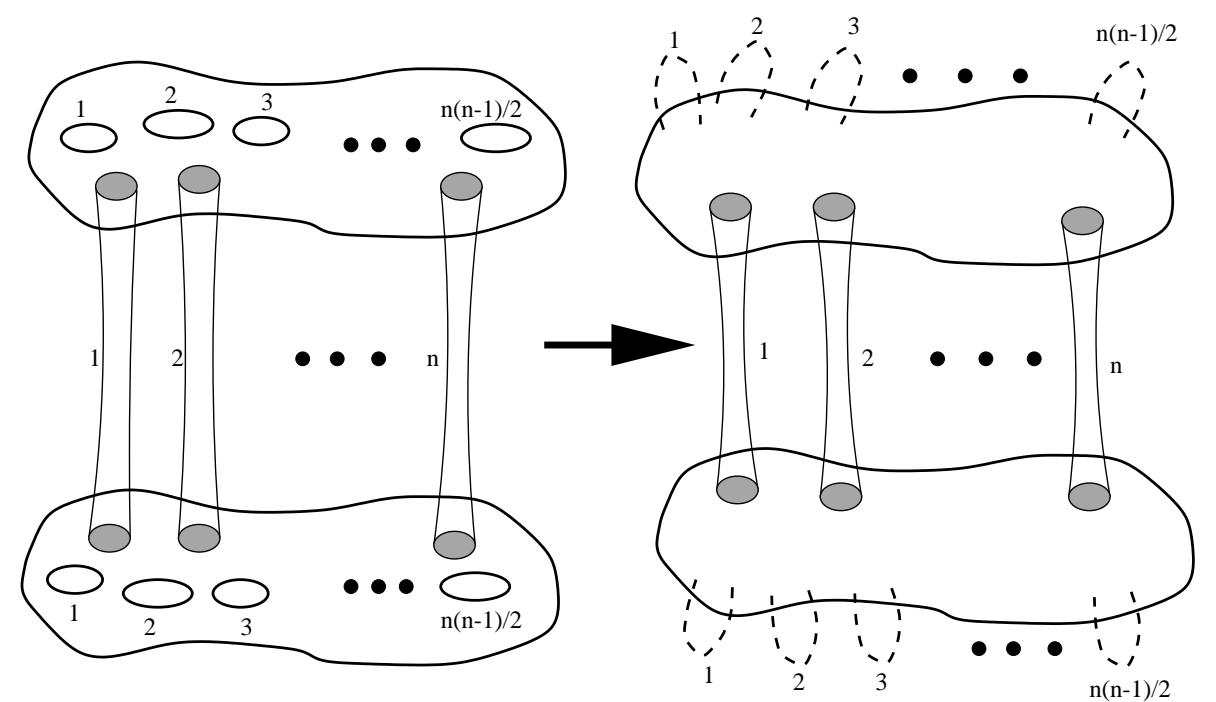

Fig. 9: The general curve (4.2) as a double cover of the curve (4.17) with a genus $g_{*}=\frac{n(n-1)}{2}$. Similarly to fig. 7 , when the curve (4.17) completely degenerates into a rational curve the curve of two matrix model (4.2) degenerates into the curve of genus $g_{\mathrm{red}}=n-1$.

where by dots we denoted monomials of lower degrees in $X$ and $Y$, and there are no "mixed" terms in the eq. (4.17). The genus of the curve (4.17) can be again easily computed by the Newton polygon, which gives

$$
g_{*}=\frac{n(n-1)}{2}
$$

In the same way one may present the generic curve of the two matrix model (4.2) as a double cover of (4.17) with $2 n$ branch points. Indeed, the Riemann-Hurwitz formula

$$
2-2 g=\# S \cdot\left(2-2 g_{0}\right)-\# B . P .
$$

where \# $S$ is number of sheets and \# B.P. is number of branch points, gives for $g=n^{2}-1$ and $g_{0}=g_{*}$ exactly $\#$ B.P. $=2 n$. It means that the generic curve of the two matrix model (4.2) can be presented as a double cover of the curve (4.17) with $n$ cuts, and when the curve (4.17) degenerates into a rational one, the curve (4.2) has the genus

$$
g_{\text {red }}=n-1
$$




\subsection{Rational degenerations}

Let us finally say a few words about the rational degenerations of (4.2), i.e. when its (smooth) genus vanishes. A particular example of such a totally degenerate curve is given by the "classical" curve (2.4), but the rational case can be easily studied for the generic values of coefficients in (4.2), i.e. without any reality restriction.

In such situation eq. (4.2) can be resolved via the (generalized) conformal map

$$
\begin{gathered}
z=r w+\sum_{k=0}^{n} \frac{u_{k}}{w^{k}} \\
\tilde{z}=\frac{r}{w}+\sum_{k=0}^{n} \bar{u}_{k} w^{k}
\end{gathered}
$$

and the substitution of (4.21) into (4.2) gives a system of equations, expressing all coefficients $f_{i j}$ in terms of parameters of the conformal map (4.21).

Indeed, substituting (4.21) into (4.2) and computing the residues one finds that the expressions

$$
R_{l}[F]=\operatorname{res}\left(\frac{d w}{w} w^{l} F(z(w), \tilde{z}(w))\right)=0
$$

for $l=-n(n+1), \ldots, n(n+1)$ form a triangular system of equations onto the coefficients $f_{i j}$. It means that each of the equations (4.22) is linear in one of the coefficients, and can be resolved step by step, starting from the ends of the chain.

For the cubic potential $(n=2)$ the solution is

$$
\begin{gathered}
a=-\frac{r^{2}}{u_{2}} \\
b=\frac{u_{1} r}{u_{2}}-2 \bar{u}_{0} \\
c=-\frac{u_{1} r \bar{u}_{0}}{u_{2}}+\bar{u}_{0}^{2}-2 r \bar{u}_{1}+3 \frac{r^{2} u_{0}}{u_{2}}-\frac{r^{3} \bar{u}_{1}}{\bar{u}_{2} u_{2}} \\
f=r^{2}-2 u_{2} \bar{u}_{2}+4 u_{0} \bar{u}_{0}+\frac{r^{4}}{\bar{u}_{2} u_{2}}-u_{1} \bar{u}_{1}-2 \frac{r \bar{u}_{1} \bar{u}_{0}}{\bar{u}_{2}}+\frac{r^{2} \bar{u}_{1} u_{1}}{\bar{u}_{2} u_{2}}-2 \frac{r u_{0} u_{1}}{u_{2}} \\
q=-3 \frac{r^{2} u_{0}^{2}}{u_{2}}+2 u_{2} \bar{u}_{2} \bar{u}_{0}-u_{2} \bar{u}_{1}^{2}-2 \frac{r^{2} \bar{u}_{1}^{2}}{\bar{u}_{2}}-\frac{r^{4} \bar{u}_{0}}{\bar{u}_{2} u_{2}}-3 r \bar{u}_{2} u_{1}+\bar{u}_{0} u_{1} \bar{u}_{1} \\
+4 u_{0} r \bar{u}_{1}-2 \bar{u}_{0}^{2} u_{0}-\frac{r \bar{u}_{1} u_{1}^{2}}{u_{2}}-r^{2} \bar{u}_{0}+2 \frac{r u_{1} \bar{u}_{0} u_{0}}{u_{2}}+3 \frac{r^{3} u_{1}}{u_{2}} \\
+\frac{r \bar{u}_{1} \bar{u}_{0}^{2}}{\bar{u}_{2}}-\frac{r^{2} \bar{u}_{0} u_{1} \bar{u}_{1}}{\bar{u}_{2} u_{2}}+2 \frac{u_{0} r^{3} \bar{u}_{1}}{\bar{u}_{2} u_{2}}
\end{gathered}
$$




$$
\begin{gathered}
h=-\frac{r^{6}}{\bar{u}_{2} u_{2}}+\frac{r^{2} \bar{u}_{0}^{3}}{\bar{u}_{2}}+\bar{u}_{2} \bar{u}_{0} u_{1}^{2}-\frac{u_{0} r \bar{u}_{1} \bar{u}_{0}^{2}}{\bar{u}_{2}}+u_{2} u_{0} \bar{u}_{1}^{2}-3 \frac{r^{3} \bar{u}_{0} \bar{u}_{1}}{\bar{u}_{2}}-\frac{\bar{u}_{2} u_{1}^{3} r}{u_{2}} \\
-\frac{u_{2} r \bar{u}_{1}^{3}}{\bar{u}_{2}}+\frac{r^{2} u_{0}^{3}}{u_{2}}+2 \frac{r^{4} \bar{u}_{1} u_{1}}{\bar{u}_{2} u_{2}}+\frac{u_{0} \bar{u}_{0} \bar{u}_{1} r^{2} u_{1}}{\bar{u}_{2} u_{2}}-\frac{r^{3} u_{1} \bar{u}_{0}^{2}}{\bar{u}_{2} u_{2}}-\frac{r^{3} \bar{u}_{1} u_{0}^{2}}{\bar{u}_{2} u_{2}}-\frac{u_{1}^{2} \bar{u}_{1}^{2} r^{2}}{\bar{u}_{2} u_{2}} \\
+3 r^{4}+\bar{u}_{0}^{2} u_{0}^{2}+\frac{u_{1}^{2} \bar{u}_{1} u_{0} r}{u_{2}}-2 \bar{u}_{0}^{2} u_{1} r+2 \frac{u_{1}^{2} r^{2} \bar{u}_{0}}{u_{2}}+\frac{u_{1} \bar{u}_{1}^{2} r \bar{u}_{0}}{\bar{u}_{2}}+r^{2} \bar{u}_{0} u_{0} \\
-u_{1} \bar{u}_{1} u_{0} \bar{u}_{0}+\frac{u_{0} \bar{u}_{0} r^{4}}{\bar{u}_{2} u_{2}}+u_{2}^{2} \bar{u}_{2}^{2}-\frac{u_{1} r \bar{u}_{0} u_{0}^{2}}{u_{2}}-2 r \bar{u}_{1} u_{0}^{2}+2 \frac{u_{0} r^{2} \bar{u}_{1}^{2}}{\bar{u}_{2}}-3 \frac{r^{3} u_{0} u_{1}}{u_{2}} \\
-\bar{u}_{1} u_{1} \bar{u}_{2} u_{2}-3 r^{2} \bar{u}_{2} u_{2}+3 u_{2} r \bar{u}_{1} \bar{u}_{0}-2 \bar{u}_{2} u_{2} \bar{u}_{0} u_{0}-\bar{u}_{1} r^{2} u_{1}+3 u_{0} r \bar{u}_{2} u_{1}
\end{gathered}
$$

together with the "complex conjugated" expressions for $\bar{a}, \bar{b}, \bar{c}$ and $\bar{q}$, where one should replace $u_{k}$ by $\bar{u}_{k}$ and vice versa. Resolving (4.22) one gets the explicit description of the rational degeneration of the curve (4.2) in terms of the coefficients of conformal map (4.21). However, in general situation they are only implicitly defined through the parameters of the potential $V(z, \bar{z})$.

\section{Quasiclassical tau-function}

Let us now define the partition function for the two matrix model (3.7) in terms of the quasiclassical tau-function introduced in [16]. First, we discuss a simpler example of the one matrix model and then turn to the particular features of the two-matrix case. The hyperelliptic curve of the one matrix model was first discussed in [17], and recently, in the most general form including all the extrema of the potential, in [19] (see also [20] and [33]).

\subsection{One-matrix model}

The complex curve of the one matrix model

$$
\mathcal{Z}=\int d \Phi e^{\operatorname{Tr} W_{n}(\Phi)}
$$

with the model potential

$$
W_{n}^{\prime}(\Phi)=\sum_{k=1}^{n} k t_{k} \Phi^{k-1}
$$

comes from the very simple loop equation $G^{2}+2 W_{n}^{\prime}(\lambda) G-f(\lambda)=0$ (see, for example, [34] or [4]). It is always hyperelliptic, i.e. can be rewritten in the form

$$
y^{2}=W_{n}^{\prime}(\lambda)^{2}+f(\lambda)
$$


with $y=G+W_{n}^{\prime}$ and the moduli hidden in the coefficients of the polynomial

$$
f(\lambda)=\sum_{k=0}^{n-1} f_{k} \lambda^{k}
$$

The generating differential is chosen as

$$
d S^{1 M M}=\frac{1}{2 \pi i} y d \lambda
$$

and additional variables, corresponding to the eigenvalue filling numbers, can be introduced through its periods

$$
S_{i}=\oint_{A_{i}} d S^{1 M M}
$$

directly related to the integrals of density over the eigenvalue supports. Then

$$
\begin{aligned}
\frac{\partial d S^{1 M M}}{\partial S_{i}} & =d \omega_{i} \\
\oint_{A_{i}} d \omega_{j} & =\delta_{i j}
\end{aligned}
$$

where the derivatives are taken at fixed coefficients $\left\{t_{l}\right\}$ of the potential $W_{n}^{\prime}(\lambda)$ (5.2). As usual, the periods dual to (5.6) are given by the integrals over dual cycles

$$
\Pi_{i}^{1 M M}=\oint_{B_{i}} d S^{1 M M} .
$$

To complete the set of parameters of the model, we have to add to the filling numbers (5.6) and coefficients of the potential (5.2) the variable

$$
\operatorname{res}_{\infty_{+}}\left(d S^{1 M M}\right)=-\operatorname{res}_{\infty_{-}}\left(d S^{1 M M}\right)=\frac{f_{n-1}}{2 t_{n}} \equiv t_{0}
$$

so that

$$
\frac{\partial d S^{1 M M}}{\partial t_{0}}=t_{n} \frac{\lambda^{n-1} d \lambda}{y}+\frac{1}{2} \sum_{k=0}^{n-2} \frac{\partial f_{k}}{\partial t_{0}} \frac{\lambda^{k} d \lambda}{y} .
$$

The dependence of $\left\{f_{k}\right\}$ with $k=0,1, \ldots, n-2$ upon $t_{0}$ is fixed by

$$
\oint_{A_{i}}\left(t_{n} \frac{\lambda^{n-1} d \lambda}{y}+\frac{1}{2} \sum_{k=0}^{n-2} \frac{\partial f_{k}}{\partial t_{0}} \frac{\lambda^{k} d \lambda}{y}\right)=0
$$

which gives for $i=1, \ldots, n-1$ exactly $n-1$ relations on $f_{0}, f_{1}, \ldots, f_{n-2}$. The bipole differential (5.10) can be also rewritten as

$$
d \Omega_{ \pm}=\frac{\partial d S^{1 M M}}{\partial t_{0}}=d \log \left(\frac{E\left(P, \infty_{+}\right)}{E\left(P, \infty_{-}\right)}\right)
$$


where $E\left(P, P^{\prime}\right)$ is the Prime form on (5.3). Obviously, the differentials (5.12) obey the properties

$$
\begin{array}{r}
\operatorname{res}_{\infty_{+}} d \Omega_{ \pm}=-\operatorname{res}_{\infty_{+}} d \Omega_{ \pm}=1 \\
\oint_{A_{i}} d \Omega_{ \pm}=0, \quad i=1, \ldots, n-1
\end{array}
$$

To complete the setup one should also add to (5.8) the following formula

$$
\Pi_{0}=\int_{\infty_{-}}^{\infty_{+}} d S^{1 M M}
$$

which can be regularized in the usual way presenting the puncture at infinity as a degenerate handle. The partition function of the multi-cut solution of the 1MM is defined now in terms of the quasiclassical tau-function $\mathcal{F}^{1 M M}$ obeying the equations [17], [19]

$$
\begin{array}{r}
\frac{\partial \mathcal{F}^{1 M M}}{\partial S_{i}^{1 M M}}=\Pi_{i}^{1 M M} \\
\frac{\partial \mathcal{F}^{1 M M}}{\partial t_{0}}=\Pi_{0}
\end{array}
$$

In the papers [19], instead of $t_{0}$ the parameter $\tilde{S}=t_{0}-\sum_{i=1}^{n-1} S_{i} \equiv S_{n}$ was used. This is a non-standard definition of homology basis on (5.3) and it gives rise to the divergences at infinities. However, the basis of [19] is related to the canonical one by a linear change of variables, where no divergences appear (except of the trivial one in (5.14)) and the integrability of (5.15) (the symmetry of second derivatives) follow from the Riemann bilinear relations, including the symmetry of period matrix of (5.3).

\subsection{Two-matrix model (general potential)}

In the same way, the filling numbers can be defined for the two matrix model

$$
S_{i}=\frac{1}{2 \pi i} \int_{i-\mathrm{th} \mathrm{spot}} d z \wedge d \bar{z}=\frac{1}{2 \pi i} \oint_{A_{i}} \tilde{z} d z=\oint_{A_{i}} d S^{2 M M}
$$

i.e. as periods of the generating differential

$$
d S^{2 M M}=\frac{1}{2 \pi i} \tilde{z} d z
$$

under the appropriate choice $\left\{A_{i}\right\}$ for the basis of $A$-cycles on the Riemann surface (4.2), (or (4.5)). This is illustrated by fig. 4 (or by its particular cubic case fig. 3), taking into account fig. 6 and eqn. (4.12). From (5.16) one still gets in the same way the analogs of the formulas (5.7)

$$
\begin{array}{r}
\frac{\partial d S^{2 M M}}{\partial S_{i}}=d \omega_{i} \\
\oint_{A_{i}} d \omega_{j}=\delta_{i j}
\end{array}
$$


where the canonical holomorphic differentials (now on the curve (4.2)) are certain linear combinations (with moduli dependent coefficients) of $g=n^{2}-1$ "lower degree" holomorphic differentials (4.4).

The derivatives of (5.17) with respect to the coefficients of the equation (4.2) can be computed in the standard way. Choosing $z$ as a covariantly constant function one writes for $(4.2)$

$$
F_{\tilde{z}} \delta \tilde{z}+\delta F=0
$$

where $\delta F \equiv \sum \delta f_{i j} z^{i} \tilde{z}^{j}$ is a variation of only the coefficients of (4.2). Then the variation of (5.17) gives rise to

$$
\delta \tilde{z} d z=-\delta F \frac{d z}{F_{\tilde{z}}}=-\sum \delta f_{i j} z^{i} \tilde{z}^{j} \frac{d z}{F_{\tilde{z}}}
$$

Expression (5.20) contains a decomposition of the variation of the meromorphic differential (5.17) over some basis of meromorphic and holomorphic differentials on the curve (4.2). It is easy to check that the coefficients $f_{i j}$ corresponding to the meromorphic Abelian differentials of the second kind can be expressed through the parameters of the potential $V(z, \bar{z})$ of the two-matrix model, namely, through the coefficients of its harmonic part $W(z)+\bar{W}(\bar{z})$. The corresponding relations follow from the fact that the complex curve (4.2) should satisfy the asymptotic expansion of the branch

$$
\tilde{z}=W^{\prime}(z)+O\left(\frac{1}{z}\right)=\sum_{k=1}^{n+1} k t_{k} z^{k-1}+O\left(\frac{1}{z}\right)
$$

which gives rise, e.g. to $\bar{a}^{-1}=-(n+1) t_{n+1}$ etc.

The rest of coefficients $f_{i j}$ consists of the coefficient corresponding to the third kind Abelian differential (with the first-order pole) and the holomorphic differentials (4.4). Fixing the coefficients expressed through the parameters of the potential in (5.20) and taking appropriate linear combinations, one arrives at (5.18).

As it will be shown below, the dependence of the free energy (3.7) upon the filling numbers (5.16) is defined by

$$
\frac{\partial \mathcal{F}}{\partial S_{i}}=\oint_{B_{i}} d S^{2 M M}=\frac{1}{2 \pi i} \oint_{B_{i}} \tilde{z} d z
$$

where $\left\{B_{i}\right\}$ are the canonical dual cycles $A_{i} \circ B_{j}=\delta_{i j}$. The integrability of (5.22) follows from the symmetry of the period matrix of the curve (4.2).

Now, as in the one matrix case, one should also introduce

$$
\operatorname{res}_{\infty_{+}}\left(d S^{2 M M}\right)=-\operatorname{res}_{\infty_{-}}\left(d S^{2 M M}\right)=t_{0}
$$


adding the bipole differential

$$
d \Omega_{ \pm}=\frac{\partial d S^{2 M M}}{\partial t_{0}}=d \log \left(\frac{E\left(P, \infty_{+}\right)}{E\left(P, \infty_{-}\right)}\right)=z^{n-1} \tilde{z}^{n-1} \frac{d z}{F_{\tilde{z}}}-\sum_{\text {holomorphic }} \frac{\partial f_{i j}}{\partial t_{0}} d v_{i j}
$$

where coefficients $\frac{\partial f_{i j}}{\partial t_{0}}$ are fixed, as in (5.11), by $\oint_{A_{i}} d \Omega_{ \pm}=0$. The variables (5.16) should be directly identified with those introduced in (3.7) for $\alpha=1, \ldots, n^{2}-1$ while $S_{n^{2}} \equiv t_{0}-\sum_{i}^{n^{2}-1} S_{i}$. The second kind Abelian differentials are defined as derivatives

$$
d \Omega_{k}=\frac{\partial d S^{2 M M}}{\partial t_{k}}, \quad d \bar{\Omega}_{k}=\frac{\partial d S^{2 M M}}{\partial \bar{t}_{k}}
$$

over the parameters of the matrix model potentials, and the dependence of the coefficients $f_{i j}$ upon the coefficients of potential is fixed by $\oint_{A_{i}} d \Omega_{k}=\oint_{A_{i}} d \bar{\Omega}_{k}=0$.

The formulas (5.16), (5.22) and (5.23), together with the (regularized) equation

$$
\frac{\partial \mathcal{F}}{\partial t_{0}}=\int_{\infty_{-}}^{\infty_{+}} d S^{2 M M}=\frac{1}{2 \pi i} \int_{\infty_{-}}^{\infty_{+}} \tilde{z} d z
$$

define the quasiclassical tau-function [16].

It is clear that this definition coincides in fact with the definition of free energy of NMM or H2MM (3.7). Indeed, using the formula (3.7) one can easily check that

$$
\frac{\partial \mathcal{F}}{\partial t_{k}}=\int z^{k} \rho(z, \bar{z}) d^{2} z=\frac{1}{2 \pi i} \operatorname{res}\left(z^{k} \tilde{z} d z\right)
$$

and

$$
\frac{\partial \mathcal{F}}{\partial S_{\alpha}}=v_{\alpha}
$$

The quantities (5.27) are in fact nothing but linear combinations of (5.22) and (5.25), like in the case of the 1MM. It can be derived by carefully treating the logarithmic integral $\int d z \wedge d \bar{z} \log \left|z-z_{\alpha}\right|$, with $z_{\alpha}$ belonging to one of the eigenvalue supports. Indeed from (3.8) one can get for $v_{\alpha}-V\left(z_{\alpha}, \bar{z}_{\alpha}\right)$

$$
\begin{aligned}
\int d z \wedge d \bar{z} \log \left|z-z_{\alpha}\right|^{2} & =\sum_{\text {spots }} \int d z \wedge d \bar{z} \log \left(z-z_{\alpha}\right)+\text { c.c. }= \\
= & \sum_{\text {boundaries }} \oint \log \left(z-z_{\alpha}\right) \bar{z} d z+\text { c.c. }=\sum_{\text {boundaries }} \oint \log \left(z-z_{\alpha}\right) \tilde{z} d z+\text { c.c. }
\end{aligned}
$$




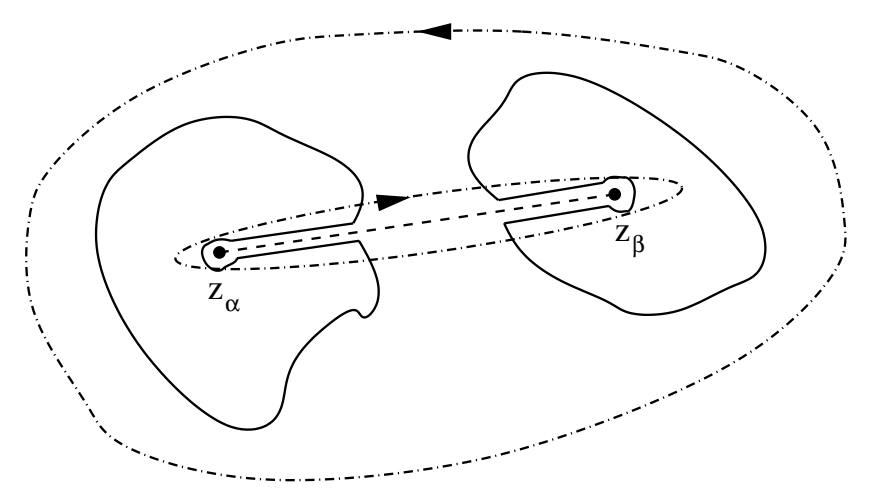

Fig. 10: The integral in (5.28) can be transformed to the (linear combinations of) the integrals over the cuts of logarithms, which turn into the B-periods of $d S^{2 M M}=\frac{1}{2 \pi i} \tilde{z} d z$.

The integral in the r.h.s. of (5.28) is an integral of the multi-valued differential defined on a Riemann surface with two sorts of cuts: the cuts of the function $\tilde{z}(z)$ and the cuts of the logarithms. The integral over the spots can be transformed into boundary integrals, where the boundaries should now include the integrals along the branches of the logarithmic cuts (see fig. 10), the corresponding contributions to the two-dimensional integrals over spots vanish. The latter ones are combined into the integrals around all spots which can be deformed to the infinity, modulo the integrals along the logarithmic cuts. Altogether these contributions gives rise to the canonical $B$-periods of $d S^{2 M M}=\frac{1}{2 \pi i} \tilde{z} d z$. Finally, the situation appears to be quite similar to 1MM since applying the procedure of "transfer" of an eigenvalue from one support to another [19] one should leave intact the boundary of the spot (since adding an eigenvalue directly to the boundary not only changes the filling numbers, but also the shape of the domain, related to the parameters of the potential [25]). Instead, as in one matrix case (where one may neglect this problem due to vanishing of the density of eigenvalues at the "boundaries" of a cut) one should put the points $z_{\alpha}$ to the branching points of the complex curve where $d z=0$. After that, using (5.28) and

$$
\left.\frac{\partial V(z, \bar{z})}{\partial z}\right|_{d z=0} \propto \oint_{c u t} \frac{\tilde{z}\left(z^{\prime}\right) d z^{\prime}}{z-z^{\prime}}
$$

one gets the formula (5.22).

Indeed, let us calculate, for example, the difference $\frac{\partial \mathcal{F}}{\partial S_{i}}-\frac{\partial \mathcal{F}}{\partial S_{j}}$ by procedure of the "eigenvalue transfer" from an endpoint $z_{\beta}=z^{\prime \prime}$ of the $\beta$-th cut to an endpoint $z_{\alpha}=z^{\prime}$ of the $\alpha$-th cut in $z$-plane. It will be given by difference of the corresponding eigenvalue effective actions (see (1.2), (1.4)) at the saddle point:

$$
\begin{array}{r}
\frac{\partial \mathcal{F}}{\partial S_{i}}-\frac{\partial \mathcal{F}}{\partial S_{j}}=-z^{\prime} \tilde{z}^{\prime}+z^{\prime \prime} \tilde{z}^{\prime \prime}+W\left(z^{\prime}\right) \\
-W\left(z^{\prime \prime}\right)+\tilde{W}\left(\tilde{z}^{\prime}\right)-\tilde{W}\left(\tilde{z}^{\prime \prime}\right)+ \\
+\sum_{m=1}^{N} \log \frac{\left(z^{\prime}-z_{m}\right)\left(\tilde{z}^{\prime}-\tilde{z}_{m}\right)}{\left(z^{\prime \prime}-z_{m}\right)\left(\tilde{z}^{\prime \prime}-\tilde{z}_{m}\right)}
\end{array}
$$


where $\tilde{z}^{\prime}=\tilde{z}\left(z^{\prime}\right), \tilde{z}^{\prime \prime}=\tilde{z}\left(z^{\prime \prime}\right)$, with the function $\tilde{z}(z)$ defined by the algebraic curve (4.2) (or (4.5)) of the two matrix model.

Passing to the continuum limit and introducing the resolvents $G(z)$ and $\tilde{G}(\tilde{z})$, as in (3.2), we rewrite the last term in (5.29) as follows

$$
\oint_{C} \frac{d z}{2 \pi i} G(z) \log \left(\frac{\left(z^{\prime}-z\right)}{\left(z^{\prime \prime}-z\right)}\right)+\oint_{\tilde{C}} \frac{d \tilde{z}}{2 \pi i} \tilde{G}(\tilde{z}) \log \left(\frac{\left(\tilde{z}^{\prime}-\tilde{z}\right)}{\left(\tilde{z}^{\prime \prime}-\tilde{z}\right)}\right)
$$

where the contours $C$ and $\tilde{C}$ encircle all the eigenvalue supports - the cuts in the $z$ and $\tilde{z}$ planes, respectively (these cuts or their stacks are depicted in fig. 5). It is important that, according to the definition of the sums in the last term of (5.29), the contours do not encircle the logarithmic cuts along $\left(z^{\prime}, z^{\prime \prime}\right)$ and $\left(\tilde{z}^{\prime}, \tilde{z}^{\prime \prime}\right)$ intervals.

Blowing up the contours $C, \tilde{C}$ we will encircle only the logarithmic cuts (note that there are no poles at infinity). Calculating discontinuity along these cuts we reduce the contour integrals to the ordinary ones:

$$
\frac{\partial \mathcal{F}}{\partial S_{i}}-\frac{\partial \mathcal{F}}{\partial S_{j}}=-z^{\prime} \tilde{z}^{\prime}+z^{\prime \prime} \tilde{z}^{\prime \prime}+\int_{z^{\prime \prime}}^{z^{\prime}} d z \tilde{z}(z)+\int_{\tilde{z}^{\prime \prime}}^{\tilde{z}^{\prime}} d \tilde{z} z(\tilde{z})
$$

the potentials being absorbed into the functions $\tilde{z}(z)=W^{\prime}(z)+G(z)$ and $z(\tilde{z})=\tilde{W}^{\prime}(\tilde{z})+$ $\tilde{G}(\tilde{z})$, according to the saddle point equations of the two matrix model.

Integrating by parts in the last term of (5.30) (note that the last integral after the change of variables goes along the unphysical sheets of the curve fig. 4) we finally get the integral over the dual $B_{i j}$ cycle

$$
\frac{\partial \mathcal{F}}{\partial S_{i}}-\frac{\partial \mathcal{F}}{\partial S_{j}}=\oint_{B_{i j}} \frac{\tilde{z} d z}{2 \pi i}
$$

which is equivalent to the equation (5.22) defining the geometry of the planar limit in two matrix model.

\subsection{Explicit form of the two-support solution for the real cubic potential}

In the rest of this section we will study in detail the degenerate case of a cubic potential with real coefficients, having only two eigenvalue supports (on the real axis), instead of four. The degeneration greatly simplifies the calculations. The period integrals can be even rewritten in terms of elliptic integrals.

Indeed, the degenerate torus (4.14) can be presented as

$$
Y^{2}+P(X)=Y^{2}-\frac{1}{g}\left(X-X_{1}\right)^{2}\left(X-X_{2}\right)=0
$$


and the new parameters $X_{1}$ and $X_{2}$ are defined by the discriminant equations

$$
\begin{aligned}
P(X) & =-\frac{1}{g} X^{3}+\left(\frac{T}{g}-\frac{9}{4 g^{2}}\right) X^{2}+\left(q+\frac{3 T}{g^{2}}-\frac{3 f}{g}\right) X+h-\frac{1}{4}\left(\frac{2 T}{g}-f\right)^{2}=0 \\
P^{\prime}(X) & =-\frac{3}{g} X^{2}+\left(\frac{2 T}{g}-\frac{9}{2 g^{2}}\right) X+\left(q+\frac{3 T}{g^{2}}-\frac{3 f}{g}\right)=0
\end{aligned}
$$

where we used (4.14) and (4.8). This system of eqs. fixes, for example, $h$ which is now not independent and can be expressed through $q$ and $f$.

Note that we have the expansions

$$
\begin{array}{r}
X_{1}=-\frac{1}{g}+\delta X_{1}=-\frac{1}{g}+O(\delta f, \delta q) \\
X_{2}=-\frac{1}{4 g}+T+\delta X_{2}=-\frac{1}{4 g}+T+O(\delta f, \delta q)
\end{array}
$$

where

$$
\begin{gathered}
\delta f=f-\frac{1}{g^{2}} \\
\delta q=q+\frac{T}{g^{2}}
\end{gathered}
$$

are deviations of moduli from their classical values in eq. (2.4). The "classical" value of $X_{1}$ in (5.34) precisely corresponds to the classical solution of (2.4), of course different from diagonal the $z=\tilde{z}$.

Substituting $z=\tilde{z}$ into eq. (4.6) one gets the fourth-order equation

$$
\left(z^{2}+\frac{3}{g} z-\frac{T}{g}+\frac{f}{2}\right)^{2}+P(2 z)=\left(z^{2}+\frac{3}{g} z-\frac{T}{g}+\frac{f}{2}\right)^{2}+\frac{1}{g}\left(2 z-X_{1}\right)^{2}\left(2 z-X_{2}\right)=0
$$

corresponding to the four branch points of two remaining cuts. These cuts can be interpreted as "splittings" of the double zeroes of the "classical limit" of this equation given by (2.6). Let us denote the splitting as $\hat{z}_{1,2} \rightarrow \hat{z}_{1,2}^{ \pm}$, so that $\hat{z}_{1}^{ \pm}$and $\hat{z}_{2}^{ \pm}$are four solutions to (5.36). Then the only non-degenerate $A$ - and $B$-periods on the torus are given by the integrals of the differential $d S^{2 M M}=\frac{1}{2 \pi i} \tilde{z} d z$ between these points

$$
\begin{array}{r}
S=\frac{1}{2 \pi i} \oint_{\hat{z}_{1}^{-}}^{\hat{z}_{1}^{+}} \tilde{z} d z \\
\frac{\partial \mathcal{F}^{2 M M}}{\partial S}=\Pi=\frac{1}{2 \pi i} \oint_{\hat{z}_{1}^{+}}^{\hat{z}_{2}^{-}} \tilde{z} d z=\frac{1}{\pi i} \int_{\hat{z}_{1}^{+}}^{\hat{z}_{2}^{-}} \tilde{z} d z
\end{array}
$$

where $\tilde{z}$ is related to $z$ via

$$
\left(z \tilde{z}+\frac{3}{2 g}(z+\tilde{z})-\frac{T}{g}+\frac{f}{2}\right)^{2}+\frac{1}{g}\left(z+\tilde{z}-X_{1}\right)^{2}\left(z+\tilde{z}-X_{2}\right)=0 .
$$


These relations for the only nontrivial period $S(5.37)$ on this degenerate $g_{\text {red }}=1$ curve should be supplemented by the relations (5.23) and (5.25), defining the dependence on $t_{0}$. As usual, one may choose instead their linear combinations $S_{1}=S$ and $S_{2}=t_{0}-S$, corresponding to the filling numbers of the two cuts.

In this way we formulated the explicit solution of the two support two matrix model with the real cubic potential. We point here out again that the integrals (5.37) can be in principle calculated in terms of elliptic functions.

\section{Connection with SUSY gauge theories}

Recently it was proposed in [19] to build the geometries of underlying string theories for certain $\mathcal{N}=1$ SUSY gauge theories by effectively reducing them to the complex curves (5.3) of 1MM. The curves of the 1MM belong to the same class as the Seiberg-Witten curves of the $S U(n) \mathcal{N}=2$ SUSY gauge theories with $n-1$ fundamental matter multiplets. The understanding of this proposal directly from the field theory was considerably advanced in [35], and later in [36]. Though the parallels between matrix models and four-dimensional supersymmetric gauge theories based on the similarity of their integrable structures were noticed much earlier [13], the recent observation of [19] contains a direct conjecture relating the superpotentials in $\mathcal{N}=1$ four dimensional theories to the partition functions of the multi-cut solutions, like ones considered in our paper.

According to the proposal of [19] the effective potentials of gaugino condensates $S_{i}=\left\langle\operatorname{Tr} W_{\alpha}^{(i)} W_{(i)}^{\alpha}\right\rangle$ in a large class of four-dimensional $\mathcal{N}=1$ gauge theories can be calculated in terms of the planar limit of matrix integrals. For the $\mathcal{N}=1$ theory with one adjoint matter multiplet (broken $\mathcal{N}=2$ supersymmetric theory) the calculation reduces to the large $N$ solution of the one-matrix integral (5.1), in general having multiple cuts, as described in the previous section. When the $U(N)$ gauge group is broken to $U\left(N_{1}\right) \times U\left(N_{2}\right) \times \ldots \times U\left(N_{k}\right)$, with the classical VEV's of different subgroups located at the different extrema of the tree superpotential, the matrix model predicts the values of $k$ gaugino condensates $S_{i}=\left\langle\operatorname{Tr} W_{\alpha}^{(i)} W_{(i)}^{\alpha}\right\rangle$ corresponding to the the vector multiplets $W_{\alpha}^{(i)}$ of the gauge subgroups. The effective potential $W_{\text {eff }}\left(S_{i}, \tau\right)$ as a function of these condensates and the complexified gauge coupling $\tau$ can be related to the free energy of the multi-cut solution as follows

$$
W_{e f f}(S, \tau)=\sum_{i} N_{i}\left(2 \pi i \tau S_{i}-\frac{\partial \mathcal{F}\left(S_{1}, \ldots, S_{k}\right)}{\partial S_{i}}\right),
$$

the logarithmic term being hidden in the second term representing the derivative of the matrix model free energy, according to the proposal of [19]. The variables $S_{1}, \ldots, S_{k}$ appear, strictly speaking, only in the planar limit of this matrix model and correspond 
to the eigenvalue filling numbers $S_{i}=\hbar N_{i} \propto N_{i} / N$ of various classical extrema of of the matrix action, giving rise to the dependence of multi-support solutions upon the variables (5.6), (5.16) discussed in our paper.

For the one-matrix model (5.1) the situation looks to be relatively simple since the key observation comes from the fact, that from the coincidence of the matrix model and Seiberg-Witten curves it trivially follows that

$$
\frac{\partial^{2} \mathcal{F}^{1 M M}}{\partial S_{i} \partial S_{j}}=\frac{\partial^{2} \mathcal{F}^{S W}}{\partial a_{i} \partial a_{j}}
$$

due to coincidence (after fixing the homology basis) of the period matrices. The SeibergWitten prepotential $\mathcal{F}^{S W}$ [12] as a function of a different set of variables

$$
a_{i}=\oint_{A_{i}} \lambda\left(\frac{d W_{n}^{\prime}}{y}-\frac{W_{n}^{\prime}}{y} \frac{d f}{2 f}\right)
$$

is defined as

$$
\frac{\partial \mathcal{F}^{S W}}{\partial a_{i}}=\oint_{B_{i}} \lambda\left(\frac{d W_{n}^{\prime}}{y}-\frac{W_{n}^{\prime}}{y} \frac{d f}{2 f}\right),
$$

and it is also a quasiclassical tau-function [11. Note that eq. (6.2) states only that the second derivatives of different functions in different variables coincide, but these functions themselves are certainly different quasiclassical tau-functions. Such identification became possible first of all since the number of multicut variables for the $1 \mathrm{MM}$ is $n-1=\operatorname{rank} S U(n)$, what is precisely the dimension of the moduli space of (the Coulomb phase of) the $S U(n)$ gauge theory.

For the softly broken $\mathcal{N}=4$ theory, according to the proposal of [19], $\mathcal{F}\left(S_{1}, \ldots, S_{k}\right)=$ $\log \mathcal{Z}$ should be calculated as the planar limit of the matrix integral

$$
\mathcal{Z}=\int \mathcal{D} \Phi_{1} \mathcal{D} \Phi_{2} \mathcal{D} \Phi_{3} e^{\operatorname{Tr}\left(i \Phi_{1}\left[\Phi_{1}, \Phi_{2}\right]+V\left(\Phi_{1}, \Phi_{2}, \Phi_{3}\right)\right)}
$$

with $\Phi_{1}, \Phi_{2}, \Phi_{3}$ considered as simple hermitean $N \times N$ matrices. Of course not all matrix integrals of this kind are calculable. An important case corresponding to the $\mathcal{N}=1^{*}$ perturbation $V\left(\Phi_{1}, \Phi_{2}, \Phi_{3}\right)=m \sum_{i=1}^{3} \Phi_{i}^{2}$ is considered in [19] and based on the planar solution of this matrix model given in [37].

If only two out of three masses are nonzero and equal, the theory possesses $\mathcal{N}=2$ supersymmetry and its non-perturbative solution is formulated in terms of the elliptic Calogero-Moser system [38], [39], [13], whose spectral curve [40] covers $n$ times the elliptic curve. There seems yet to be no naive and direct relations between the corresponding Seiberg-Witten theory and quasiclassical tau-function of two matrix model, considered in this paper. However, the structure of its complex curve suggests that certain geometric parallels between these two theories are quite possible. 
Let us discuss a possible place of the two matrix models in the context of the proposal of [19]. An obvious and interesting generalization of the $\mathcal{N}=1$ SYM theory with one adjoint chiral multiplet is the the case of a few multiplets with direct interactions among the fields. If one takes, in the case of two adjoint chiral multiplets $X$ and $Y$, the tree superpotential $W_{\text {tree }}=-\operatorname{Tr} X Y+\operatorname{Tr} W(X)+\operatorname{Tr} \tilde{W}(Y)$ then the function $\mathcal{F}\left(S_{1}, S_{2}, \ldots\right)$ in the general formula (6.1) should be chosen as the planar free energy of the two matrix model with (in general) multiple supports, being calculated according to the equations (5.16) and (5.22).

In this way we establish the relation between the algebraic curve of the two matrix model and geometry of the supersymmetric theory with the described class of tree potentials.

The two matrix models obeys a rich phase structure in the space of its couplings. Its critical points correspond to collisions of various singularities on the algebraic curve, like the collapse of the B-period of the Seiberg-Witten curve corresponding to the appearence of massless monopoles. These critical points were used for the complete classification (within the H2MM) of the models of $(p, q)$ rational matter fields interacting with 2D gravity (see for example [6]). It would be interesting to study the consequences of this well established picture for the phase structure of the underlying $\mathcal{N}=1 \mathrm{SYM}$ theory with two adjoint chiral multiplets.

Let us also note here that an interesting generalisation of the standard H2MM considered in this paper is the model describing the perturbed quantum mechanics of the inverted oscillator, proposed in [41]. The model is related to the dynamics of windings in the compactified $2 \mathrm{D}$ string theory. For rational $R$ it can be also described by an algebraic curve.

In contrast to the one matrix model, in the two matrix model the number of multicut parameters (4.3) grows exactly as the dimension of the $S U(n)$ group $n^{2}-1=\operatorname{dim} S U(n)$. Naively, this would correspond to the total breaking of the $S U(n)$ gauge group in the Seiberg-Witten like context, including even the breaking of the corresponding global symmetry, or to a theory with a more complicated gauge/matter structure.

However, an important particular case of the multicut solution of the two-matrix model corresponding to the case of real couplings in the potential and only $n-1$ real eigenvalue supports, giving the curve with genus (4.20). In this case we have the number of extra parameters exactly equal to the genus $g_{\text {red }}=n-1=\operatorname{rank} S U(n)$, which might be more appropriate for the study of the symmetry breaking in the corresponding $\mathcal{N}=1$ SYM theory.

There exist a few obvious generalizations of the multi-matrix interactions, giving rise to a very diverse class of the corresponding multi-field interactions in the $\mathcal{N}=1 \mathrm{SYM}$ theories. A rather general class of interesting multi-matrix models with tree-like interactions will be studied in the next section. 


\section{Possible generalizations}

A large class of solvable matrix models 11 can be classified by "tree diagrams", where each edge of the tree connects two matrices sitting at the vertices. The corresponding matrix model potential can be written as

$$
V(\Phi)=\operatorname{Tr}\left(-\sum_{\mathrm{i}>\mathrm{j}=1}^{Q} C_{i j} \Phi_{i} \Phi_{j}+\sum_{\mathrm{i}} W_{i}\left(\Phi_{i}\right)\right)
$$

where $C_{i j}=1$, if $i, j$ are the neighboring vertices of the tree, and $C_{i j}=0$ otherwise. A particular kind of such a solvable model with tree-like interaction, the Potts model on planar graphs, was first considered in [42].

It is easy to integrate out the "angular" parts of hermitean matrices $\Phi_{i}, \quad i=1, \ldots, Q$, since they are independent in the case of a tree interaction, taking the corresponding HCIZ integrals and to rewrite the partition function of the model in terms of their eigenvalues $\Phi_{i}=\operatorname{diag}\left(z_{1}^{(i)}, \ldots, z_{N}^{(i)}\right)$ (see for example [43])

$$
Z=\int \prod_{k=1}^{N}\left(\prod_{i}\left(d z_{k}^{(i)} e^{W_{i}\left(z_{k}^{(i)}\right)}\right)\right) e^{-\sum_{i, j} C_{i j} z_{k}^{(i)} z_{k}^{(j)}} \prod_{i=1}^{Q}\left[\Delta\left(z^{(i)}\right)\right]^{2-m_{i}}
$$

where $m_{i}=\sum_{j=1}^{Q} C_{i j}$ is the coordination number of the $i$-th vertex.

Introducing the resolvents of matrices $G_{i}(z)$ (having as usual, the assymptotics $G_{i}(z) \rightarrow t_{0} / z$ at $\left.z \rightarrow \infty\right)$ we can write down the following saddle point equations, generalizing the eqs. (3.3) of the H2MM:

$$
\sum_{j=1}^{Q} C_{i j} z^{(j)}-W_{i}^{\prime}\left(z^{(i)}\right)=\left(m_{i}-2\right) G_{i}\left(z^{(i)}\right)
$$

As in the case of the two matrix model, this system of equations should be degenerate, and this degeneracy is the condition of its solution in terms of an algebraic hyper-surface relating all $Q$ variables 12 . Namely, it should exist a polynomial function of $F$ depending on all $z^{(i)}, i=1, \ldots, Q$, such that

$$
F\left(z^{(1)}, \ldots, z^{(Q)}\right)=0
$$

in analogy with (4.1). If the system (7.3) was not degenerate it would give only pointlike distributions, leading to the collaps of eigenvalues into one or a few points.

11 where by "solvability" we mean a possibility to reduce the number of degrees of freedom from the order of $N^{2}$ to the order of $N$, by integrating over the angular variables of the matrices

12 An important paricular case of such a surface will be considered in 44. 
To build the function (7.4), and to analyse the structure of the corresponding algebraic surface we should start as usual, from the "classical" equations (cf. with (2.2)) corresponing to putting all the r.h.s. of (7.3) to zero. The "classical" limit of the function (7.4) corresponds to the product of all these "classical" equations (in analogy with (2.4) for the two matrix model). Then one can write:

$$
F\left(z^{(1)}, \ldots, z^{(Q)}\right)=\prod_{i=1}^{Q}\left[\sum_{j=1}^{Q} C_{i j} z^{(j)}-W_{i}^{\prime}\left(z^{(i)}\right)\right]+\text { deformations }=0,
$$

where by the deformations we mean adding a polynomial in all variables of lower degree, governed by corresponding multidimensional Newton polyhedron. The coefficients in front of the monomials of higher degrees are determined by the assymptotics at infinities following from (7.3) and coincide with their values in the classical part. The rest of the deformation coefficients will provide the new moduli of the complex structure of this algebraic manifold.

The algebraic equation (7.4) has the degree $\operatorname{deg}=\prod_{i=1}^{Q}\left(K_{i}-1\right)$, where $K_{i}$ is the highest power of the potential $W_{i}(z)$. This corresponds to the number of extrema in the classical multi-matrix potential and to the number of moduli parameters of the curve contained in the deformation.

The fact that this algebraic surface is in general not a curve does not contradict the existence of the resolvents $G_{i}(z)$, which means that for any two variables $z^{(i)}, z^{(j)}$ it should exist an algebraic curve $F_{i j}\left(z^{(i)}, z^{(j)}\right)=0$ with a polynomial function $F_{i j}(x, y)$ of the same degree. This loss of information in the abovementioned surface might be related to the existence of higher dimensional holomorphic differential forms, like the well known threeform $d \Omega_{3}$ on the Calabi-Yau 3-folds. On particular 3-cycles, after the integration over two variables it turns into a meromorphic one-differential. This differential is related to the resolvent with respect to the third variable [45]. In principle, we could try to restore the individual algebraic curves out of the general surface by excluding the variables one by one from the equation for the surface, using (7.3).

Of course it is only a sketch of the construction of the algebraic curve of the tree-like multi-matrix model. It would be interesing to precise the details and the structure of these algebraic curves, although it might be difficult to do it to the same extent of explicitness as we have done in this paper for the two matrix model.

Some further generalisations are possible if we substitute the potential (7.1) by

$$
V(\Phi)=\operatorname{Tr}\left(-\sum_{\mathrm{i}>\mathrm{j}=1}^{Q} C_{i j}\left(\Phi_{i} \Phi_{j}\right)+\sum_{\mathrm{i}} W_{i}\left(\Phi_{i}\right)\right)
$$


where $C_{i j}(M)$ are arbitrary polynomial functions (nonzero only on a tree). In this case we can achieve the reduction to the eigenvalues by the method of character expansion (see [43] and references therein). Namely, we can expand the exponent of each interaction term into the $G L(N)$ characters $\chi_{R}(M)$

$$
e^{-C_{i j} \operatorname{Tr}\left(\Phi_{i} \Phi_{j}\right)}=\sum_{R} f_{R}^{(i, j)} \chi_{R}\left(\Phi_{i} \Phi_{j}\right)
$$

and then use the orthogonality property of the matrix elements to integrate out the relative angle of two matrices. An example of such calculation was done in [46] for the $\operatorname{Tr}\left(\Phi_{i} \Phi_{j}\right)^{2}$ interaction. The corresponding system of polynomial equations will include, along with the eigenvalue variables, the dual variables corresponding to the highest weights of the Young tableaux of the $S L(N)$ irreducible representations. Here the construction of the algebraic surface looks even more difficult, but certainly not impossible. Note that the model still stays "solvable" by the character expansion method if we change the arguments of products $C_{i j}\left(\Phi_{i} \Phi_{j}\right)$ by $C_{i j}\left(\Phi_{i}^{k_{i j}} \Phi_{j}^{n_{i j}}\right)$ with arbitrary integers $k_{i j}, n_{i j}$ for each $i j$-link.

These models (and those which can be reduced to them by introducing some gaussian matrix integrations, like in the case of the Potts model on planar graphs [42]) exhaust the list of "solvable" (i.e., reducible to the eigenvalues) multi-matrix models.

\section{Conclusion}

In this paper we studied the multi-support solutions of two matrix models and we found that they lead to the appearence of a new nontrivial one-dimensional complex geometry. The corresponding quasiclassical tau-function can be still defined in a standard way and even rather explicit formulas defining the tau-funcion can be written down.

The main problem for the multi-support solutions is nevertheless to write down explicitly the system of integrable equations which is solved by the corresponding free energy. For the one-support solutions such system necessarily includes the dispersionless Hirota equations which have a nice and well-known dispersionfull analog.

The only known analogs of the dispersionless Hirota equations for the multi-support case are the associativity or WDVV equations [47], and a wide class of their solutions is constructed on the base of quasiclassical tau-functions. The formula (4.11) in particular suggests that the quasiclassical partition function of the cubic two matrix model in the "symmetric ansatz", say with $t_{0}=0$, satisfies the WDVV equations 47, 48]. This follows from simple counting of the number of variables (six coefficients of the potential and three filling numbers altogether give the same number of free parameters as the number of critical points (4.11) for $n=2$ ) and the structure of the residue formula for this case. We mention this fact since it seems to be the only explicitly known solution to WDVV equations coming from the nonhyperelliptic curves (see [49] for a general discussion of this issue). 
In this paper we presented a two-support solution of the two matrix model for the cubic potential in a rather explicit form. Nevertheless, in principle it can be precised further. In particular, the period integrals (5.37) are elliptic (according to the structure of the underlying curve having the genus $g_{\text {red }}=1$ ) and can be in principle calculated explicitly. It might be instructive to do it and to write down explicitly their asymptotic expansion.

The multi-solutions we found are quite interesting from the point of view of statisticalmechanical models on planar graphs. An example of such model (a double-phase Ising model) is described in section 2 . It would be interesting to classify all such possible models emerging from the multi-support two matrix model.

From the point of view of the underlying $\mathcal{N}=1$ SYM theory it is very desirable to study the degenerations of a higher genus algebraic curve (with more than two cuts filled) of the two matrix model considered above and classify the emerging physical excitations (monopoles, dyons etc), by analogy to the hyperelliptic solution in (generalized) SeibergWitten picture.

Finally, the models described in section 7 should contain a much richer variety of possible algebraic surfaces describing their planar limit. They certainly deserve a considerable attention. The matrix models, due to their natural integrability properties, could give an insight into the structure of algebraic surfaces possessing interesting physical applications.

\section{Acknowledgements:}

We are indebted to M.Aganagic, S.Alexandrov, E.Brezin, A.Gorsky, S.Gukov, H.Itoyama, I.Kostov, A.Levin, A.Mironov, A.Morozov, N.Nekrasov, V.Rubtsov and P.Wiegmann for many interesting discussions and especially to R.Dijkgraaf, I.Krichever, P.Pushkar and A.Zabrodin for very helpful conversations. The work of V.K. was partially supported by European Union under the RTN contracts HPRN-CT-2000-00122 and -00131. The work of A.M. was also partially supported by RFBR grant 02-02-16496, INTAS grant 00-00334 and grant of support of scientific schools No. 00-15-96566. A.M. would like also to thank Laboratoire de Physique Théorique de l'École Normale Supérieure and Institut des Hautes Études Scientifiques for the warm hospitality. 


\section{References}

[1] C. Itzykson, J.-B. Zuber, "The planar approximation II", J. Math. Phys. 21, (1980) 411; M.-L. Mehta, "A method of integration over matrix variables", Comm. Math. Phys. 79 (1981) 327.

[2] A. Gerasimov, A. Marshakov, A. Mironov, A. Morozov and A. Orlov, "Matrix Models Of 2D Gravity And Toda Theory," Nucl. Phys. B 357, 565 (1991).

[3] V.A. Kazakov, "Ising model on dynamical planar random lattice : exact solution", Phys. Lett. A 119 (1986) 140; D.V. Boulatov, V.A. Kazakov, "The Ising model on a random planar lattice : the structure of phase transition and the exact critical exponents", Phys. Lett. B 186 (1987) 379.

[4] V.A. Kazakov, "The appearance of matter fields from quantum fluctuations of 2Dgravity", Mod. Phys. Lett. A4 (1989) 2125

[5] M.R. Douglas, "The two matrix model", Cargèse 1990, Proceedings, Random surfaces and quantum gravity, 77-83.

[6] J.-M. Daul, V.A. Kazakov, I.K. Kostov, "Rational theories of 2D gravity from the two-matrix model", Nucl. Phys. B409 (1993) 311.

[7] S. Kharchev and A. Marshakov, "On p - q duality and explicit solutions in $c \leq 12$-d gravity models," Int. J. Mod. Phys. A 10, 1219 (1995) arXiv:hep-th/9303100.

[8] M. Mineev-Weinstein, P. B. Wiegmann and A. Zabrodin, "Integrable structure of interface dynamics," Phys. Rev. Lett. 84, 5106 (2000) arXiv:nlin.si/0001007.

[9] I. K. Kostov, I. Krichever, M. Mineev-Weinstein, P. B. Wiegmann and A. Zabrodin, " $\tau$-function for analytic curves," arXiv:hep-th/0005259.

[10] V.A. Kazakov, unpublished; as cited in a footnote of [9].

[11] A.Gorsky, I.Krichever, A.Marshakov, A.Mironov and A.Morozov, "Integrability and Seiberg-Witten Exact Solution", Phys. Lett. B355 (1995) 466; hep-th/9505035.

[12] N.Seiberg and E.Witten, "Monopole Condensation, And Confinement In N=2 Supersymmetric Yang-Mills Theory", Nucl. Phys. B426 (1994) 19; hep-th/9407087.

[13] A. Gorsky and A. Marshakov, "Towards effective topological gauge theories on spectral curves," Phys. Lett. B 375, 127 (1996) arXiv:hep-th/9510224.

[14] N. A. Nekrasov, "Seiberg-Witten prepotential from instanton counting," arXiv:hepth/0206161.

[15] A. Marshakov, "Seiberg-Witten Theory And Integrable Systems," Singapore, Singapore: World Scientific (1999) 253 p.

[16] I. M. Krichever, "The tau function of the universal Whitham hierarchy, matrix models and topological field theories," Commun. Pure. Appl. Math. 47 (1992) 437, arXiv:hepth/9205110. 
[17] F. David, "Non-Perturbative Effects in Matrix Models and Vacua of Two Dimensional Gravity, Phys.Lett. B302 (1993) 403-410; hep-th/9212106; F. David, G. Bonnet, F. David, B. Eynard, "Breakdown of universality in multi-cut matrix models," J.Phys. A33 (2000) 6739.

[18] I. K. Kostov, "Conformal field theory techniques in random matrix models," arXiv:hep-th/9907060.

[19] R. Dijkgraaf, C. Vafa, "Matrix Models, Topological Strings, and Supersymmetric Gauge Theories," hep-th/0206255; "On Geometry and Matrix Models," hep-th/0207106; "A Perturbative Window into Non-Perturbative Physics," hepth/0208048.

[20] R. Dijkgraaf, S. Gukov, V. A. Kazakov and C. Vafa, "Perturbative analysis of gauged matrix models," arXiv:hep-th/0210238.

[21] N. Dorey, T. J. Hollowood, S. P. Kumar and A. Sinkovics, "Massive vacua of N = 1* theory and S-duality from matrix models," hep-th/0209099.

[22] L. Chekhov and A. Mironov, "Matrix models vs. Seiberg-Witten/Whitham theories," hep-th/0209085.

[23] Ling-Lie Chau, O. Zaboronsky, "On the structure of Normal Matrix Model", Commun.Math.Phys. 196 (1998) 203-247, hep-th/9711091

[24] P. B. Wiegmann and A. Zabrodin, "Conformal maps and dispersionless integrable hierarchies," Commun. Math. Phys. 213, 523 (2000) arXiv:hep-th/9909147.

[25] A. Marshakov, P. Wiegmann and A. Zabrodin, "Integrable structure of the Dirichlet boundary problem in two dimensions," Commun. Math. Phys. 227, 131 (2002) arXiv:hep-th/0109048.

[26] G. Akemann, "Higher genus correlators for the Hermitian matrix models with multiple cuts", Nucl. Phys. 482, 403 (1996), hep-th/9606004.

[27] B. Eynard, "Large N expansion of the 2-matrix model," arXiv:hep-th/0210047.

[28] I.K. Kostov, "Gauge invariant matrix model for the A-D-E closed strings," Phys. Lett. B 297 (1992) 74, hep-th/9208053.

[29] A. Marshakov, A. Mironov and A. Morozov, "Generalized matrix models as conformal field theories: Discrete case," Phys. Lett. B 265, 99 (1991); S. Kharchev, A. Marshakov, A. Mironov, A. Morozov and S. Pakuliak, "Conformal matrix models as an alternative to conventional multimatrix models," Nucl. Phys. B 404, 717 (1993) arXiv:hep-th/9208044.

[30] M. Staudacher, "Combinatorial solution of the two matrix model," Phys. Lett. B 305, 332 (1993) arXiv:hep-th/9301038.

[31] D. V. Boulatov, "Infinite tension strings at $d>1$," Mod. Phys. Lett. A 8, 557 (1993), hep-th/9211064.

[32] I.Krichever, 2000, unpublished. 
[33] F. Ferrari, "Quantum parameter space and double scaling limits in N=1 super YangMills theory", hep-th/0211069.

[34] A. A. Migdal, "Loop Equations And 1/N Expansion," Phys. Rept. 102, 199 (1983).

[35] R. Dijkgraaf, M.T. Grisaru, C.S. Lam, C. Vafa, D. Zanon "Perturbative Computation of Glueball Superpotentials", hep-th/0211017.

[36] F. Cachazo, M. R. Douglas, N. Seiberg, E. Witten, "Chiral Rings and Anomalies in Supersymmetric Gauge Theory", hep-th/0211170.

[37] V. A. Kazakov, I. K. Kostov and N. A. Nekrasov, "D-particles, matrix integrals and KP hierarchy," Nucl. Phys. B 557, 413 (1999) hep-th/9810035; J. Goldstone, unpublished; J. Hoppe, MIT PhD thesis, 1982.

[38] R. Donagi and E. Witten, "Supersymmetric Yang-Mills Theory And Integrable Systems," Nucl. Phys. B 460, 299 (1996) arXiv:hep-th/9510101.

[39] E. J. Martinec, "Integrable Structures in Supersymmetric Gauge and String Theory," Phys. Lett. B 367, 91 (1996) arXiv:hep-th/9510204].

[40] I.Krichever, Func. Anal. \& Appl., 14 (1980) 282.

[41] S.Yu. Aleksandrov, V.A. Kazakov, I.K. Kostov, "Time-dependent backgrounds of 2D string theory", Nucl.Phys. B640:119-144,2002) 119-144; hep-th/0205079.

[42] V.A. Kazakov, "Exactly solvable Potts models, bond and tree-like percolation on dynamical (random) planar lattice", Nucl. Phys. B 4 (Proc. Supp.) (1988) 93.

[43] V.A. Kazakov, "Solvable matrix models", Random Matrices and their Applications, MSRI publications, volume 40 (2001), hep-th/0003064.

[44] F. Cachazo, R. Dijkgraaf, S. Gukov, V. A. Kazakov and C. Vafa, to appear.

[45] A. Klemm, W. Lerche, P. Mayr, C. Vafa, N. Warner, "Self-Dual Strings and N=2 Supersymmetric Field Theory", Nucl.Phys. B477 (1996) 746-766, hep-th/9604034.

[46] V.A.Kazakov, P. Zinn-Justin, "Two-matrix model with BABA interaction", preprint hep-th/9808043, Nucl.Phys. B546 (1999) 647-668.

[47] E. Witten, "On the structure of the topological phase of two-dimensional gravity", Nucl. Phys. B340 (1990) 281; R. Dijkgraaf, H. Verlinde and E. Verlinde, "Topological strings in $D<1$ ", Nucl. Phys. B352 (1991) 59.

[48] A. Marshakov, A. Mironov and A. Morozov, "WDVV-like equations in N=2 SUSY Yang-Mills Theory", Phys. Lett. B389 (1996) 43, hep-th/9607109.

[49] A. Marshakov, "On associativity equations," Theor. Math. Phys. 132, 895 (2002) [Teor. Mat. Fiz. 132, 3 (2002)] arXiv:hep-th/0201267. 